AIP Applied Physics

\title{
Effect of doping on polarization profiles and switching in semiconducting ferroelectric thin films
}

Vivek B. Shenoy, Yu Xiao, and Kaushik Bhattacharya

Citation: J. Appl. Phys. 111, 084105 (2012); doi: 10.1063/1.3702849

View online: http://dx.doi.org/10.1063/1.3702849

View Table of Contents: http://jap.aip.org/resource/1/JAPIAU/v111/i8

Published by the American Institute of Physics.

\section{Related Articles}

Switching performance of a polymer-stabilized antiferroelectric liquid crystal based on bent-core molecules Appl. Phys. Lett. 100, 223301 (2012)

Switching performance of a polymer-stabilized antiferroelectric liquid crystal based on bent-core molecules APL: Org. Electron. Photonics 5, 120 (2012)

Observation of room temperature saturated ferroelectric polarization in Dy substituted $\mathrm{BiFeO} 3$ ceramics J. Appl. Phys. 111, 074105 (2012)

Strain effect on the surface potential and nanoscale switching characteristics of multiferroic BiFeO3 thin films Appl. Phys. Lett. 100, 132907 (2012)

Elimination of domain backswitching in $\mathrm{BiFe} 0.95 \mathrm{Mn} 0.05 \mathrm{O} 3$ thin films by lowering the layer thickness J. Appl. Phys. 111, 066107 (2012)

\section{Additional information on J. Appl. Phys.}

Journal Homepage: http://jap.aip.org/

Journal Information: http://jap.aip.org/about/about_the_journal

Top downloads: http://jap.aip.org/features/most_downloaded

Information for Authors: http://jap.aip.org/authors

\section{ADVERTISEMENT}

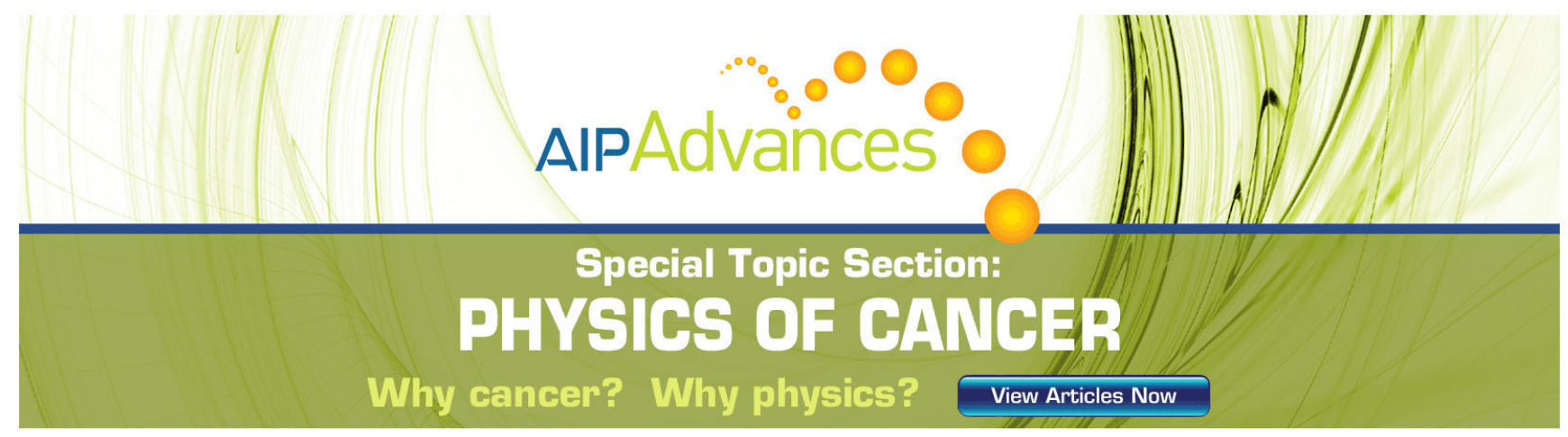




\title{
Effect of doping on polarization profiles and switching in semiconducting ferroelectric thin films
}

\author{
Vivek B. Shenoy, ${ }^{1, a)}$ Yu Xiao, ${ }^{2, b)}$ and Kaushik Bhattacharya ${ }^{2, c)}$ \\ ${ }^{1}$ School of Engineering, Brown University, Providence, Rhode Island 02912, USA \\ ${ }^{2}$ Division of Engineering and Applied Science, California Institute of Technology, Pasadena, \\ California 91125, USA
}

(Received 13 June 2011; accepted 10 March 2012; published online 19 April 2012)

\begin{abstract}
This paper proposes a theory to describe the polarization and switching behavior of ferroelectrics that are also wide-gap semiconductors. The salient feature of our theory is that it does not make any a priori assumption about either the space charge distribution or the polarization profile. The theory is used to study a metal-ferroelectric-metal capacitor configuration, where the ferroelectric is n-type doped. The main result of our work is a phase diagram as a function of doping level and thickness that shows different phases, namely, films with polarization profiles that resemble that of undoped classical ferroelectrics, paraelectric, and a new head-to-tail domain structure. We have identified a critical doping level, which depends on the energy barrier in the Landau energy and the built-in potential, which is decided by the electronic structures of both the film and the electrodes. When the doping level is below this critical value, the behavior of the films is almost classical. We see a depleted region, which extends through the film when the film thickness is very small, but is confined to two boundary layers near the electrodes for large film thickness. When the doping level is higher than the critical value, the behavior is classical for only very thin films. Thicker films at this doping level are forced into a tail-to-tail configuration with three depletion layers, lose their ferroelectricity, and may thus be described as nonlinear dielectric or paraelectric. For films which are doped below the critical level, we show that the field required for switching starts out at the classical coercive field for very thin films, but gradually decreases. (C) 2012 American Institute of Physics. [http://dx.doi.org/10.1063/1.3702849]
\end{abstract}

\section{INTRODUCTION}

Ferroelectric perovskite thin films used in device and memory applications are often modeled following the classical Devonshire-Ginzburg-Landau theory as insulators. These materials, however, are wide bandgap semiconductors. Consequently, one has band-bending near the electrodes, depletion layers, and the formation of Schottky barriers. ${ }^{1}$ The polarization profiles can also be altered. Significantly, these and many resulting properties of the ferroelectric films depend on dopant concentration and the density of crystalline defects, such as oxygen vacancies.

The study of the coupling of polarization and space charges in ferroelectrics has a long history-see, for example, Fridkin ${ }^{2}$ and the literature review in Bratkovsky and Levanyuk. ${ }^{3}$ Various technological applications, including ferroelectric memories, very thin ferroelectric films and optical fixing of domain walls, has led to a renewed interest in the subject. Tagantsev et $a{ }^{4}{ }^{4}$ assumed the existence of a depleted layer of a given thickness $W$ (which is treated as a phenomenological parameter) and studied its effect on switching and on fatigue. They showed that their results agreed qualitatively with experimental observations. Bratkovsky and Levanyuk ${ }^{3}$ as well as Pintilie and Alexe ${ }^{5}$ assume a priori a distribution of space charges and study its effect

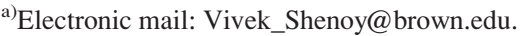

${ }^{b}$ Present address: Corning Inc., Corning, NY 14831, USA.

${ }^{c)}$ Electronic mail: bhatta@its.caltech.edu.
}

on the ferroelectric phase transition. Watanabe $e^{6,7}$ treats the ferroelectric as a semiconductor, but assumes that the polarization is uniform through the thickness of a thin film. Zubko et al. ${ }^{8}$ modified the coefficients of the Devonshire-Ginzburg-Landau theory and introduced the notion of effective polarization to account for space charges. Recently, Baudry, ${ }^{9}$ using a discrete approach, as well as Morozovska et al., ${ }^{10}$ extending the Devonshire-Ginzburg-Landau, have developed a coupled field theory of space charges and polarization, but make a priori assumptions on the space charge distribution when studying specific problems. Gureev et al. ${ }^{11}$ have studied head-to-head domain walls, while Eliseev et al. ${ }^{12}$ and Maksymovych et al. ${ }^{13}$ have studied static and dynamic conductivity of domain walls. All of these studies have provided a number of insights into how space charges alter polarization behavior. Still, a comprehensive study that makes no $a$ priori assumptions on the polarization and space charge is missing.

In this paper, we adapt the Devonshire-Ginzburg-Landau theory to ferroelectrics, which are also wide bandgap semiconductors. This theory does not make any a priori assumption about either the space charge distribution or the polarization profile. We study a metal-ferroelectric-metal capacitor configuration. Ferroelectric perovskites are typically p-type semiconductors in bulk, if undoped. However, n-type inversion layers are observed in thin films near the surfaces. And it is also possible to prepare n-type ferroelectric perovskites in bulk by growing the specimen in an oxygen-poor atmosphere. ${ }^{1}$ Since we are mainly interested in thin films, for 
definiteness, we assume that the ferroelectric is n-type doped and that the concentration of acceptor impurities is negligible. We could proceed analogously for p-type ferroelectrics. We show that the polarization profile and the space charge distribution can be quite complicated, depending on the dopant concentration and on the thickness of the film. We provide a detailed characterization of the profiles for different dopant concentration and thicknesses.

The current paper limits itself to equilibria as static loads or loads that change slowly with respect to the relaxation times. An extension of this theory to a time-dependent situation is presented in Suryanarayana and Bhattacharya. ${ }^{14}$ An early report of this current work was presented in Xiao et al., ${ }^{15}$ and a detailed mathematical derivation of the model with deformation is described in Xiao and Bhattacharya. ${ }^{16}$

The paper is organized as follows: We present the theory in Sec. II and describe some computational results in Sec. III. Section IV presents an approximate analytical model, which allows us to very easily and explicitly calculate the polarization profile, the space-charge distribution and the electric potential. We show that this approximate model is very accurate by comparing these with detailed computations. We conclude in Sec. V.

\section{THE MODEL}

Consider a film of thickness $L$ with metal electrodes on the two surfaces. The total potential energy per unit area of the film for a polarization field $p(x)$ and charge distribution $\rho(x)$ is given by

$$
\begin{aligned}
E[p, \rho]= & \int_{0}^{L}\left(\frac{\kappa(x)}{2}\left(\frac{d p}{d x}\right)^{2}+W(p, x)+W_{\rho}(\rho, x)\right. \\
& \left.+\frac{\epsilon_{0}}{2}\left(\frac{d \phi}{d x}\right)^{2}\right) d x-\sigma_{0} \phi_{0}-\sigma_{L} \phi_{L},
\end{aligned}
$$

where $\phi$ is the electric potential obtained by solving Maxwell's equation,

$$
\frac{d}{d x}\left(-\epsilon_{0} \frac{d \phi}{d x}+p\right)=\rho
$$

on $(0, L)$ subject to the boundary conditions

$$
\phi(0)=\phi_{0}, \quad \phi(L)=\phi_{L}
$$

and $\sigma_{0}, \sigma_{\mathrm{L}}$ are the free charges that are induced on the ferroelectric/metal boundary by the applied voltage boundary condition,

$$
\sigma_{0}=\left(-\epsilon_{0} \frac{d \phi}{d x}=p\right)_{x=0}, \quad \sigma_{L}=\left(\epsilon_{0} \frac{d \phi}{d x}-p\right)_{x=L}
$$

The terms of the potential energy may be understood as follows: The first term penalizes rapid changes in polarization and is related to the energy in forming a domain wall. The coefficient $\kappa$ is positive. The second is the DevonshireLandau energy, which carries the information about the spontaneous polarized state. It depends on temperature; how- ever, this dependence has not been shown explicitly since we shall only be interested in an isothermal situation. The third term is the potential energy associated with injecting/depleting charges in semiconductors. Notice that we have allowed the first three terms to explicitly depend on position, as, for example, in a heterogeneous or heterogeneously doped film. The fourth term is the energy associated with the total electric field (applied and induced). The last two terms is the work done by the battery in maintaining the applied potentials.

We seek stable equilibrium states— $p(x), \rho(x)$ —of this potential energy. To derive the equilibrium equation, we take the first variation,

$$
\delta E=\left.\frac{d}{d \eta} E[p+\eta q, \rho+\eta \xi]\right|_{\eta=0}=0
$$

for some $q, \xi$. To evaluate this for some given $q$ and $\xi$, let $\psi$ solve the Maxwell's equation,

$$
\frac{d}{d x}\left(-\epsilon_{0} \frac{d \psi}{d x}+q\right)=\xi
$$

subject to $\psi(0)=\psi(L)=0$, and let $\zeta_{0}, \zeta_{L}$ be the induced charges defined analogously to Eq. (4). We may regard $\psi$ as the variation in the electric potential induced by the variation in polarization and charge. It follows

$$
\begin{aligned}
\delta E= & \int_{0}^{L}\left(\kappa \frac{d p}{d x} \frac{d q}{d x}+\frac{\partial W}{\partial p} q+\frac{\partial W_{\rho}}{\partial \rho} \xi+\epsilon_{0} \frac{d \phi}{d x} \frac{d \psi}{d x}\right) d x \\
& -\zeta_{0} \phi_{0}-\zeta_{L} \phi_{L}=0 .
\end{aligned}
$$

However,

$$
\begin{aligned}
\int_{\Omega} \kappa \frac{d p}{d x} \frac{d q}{d x} d x & =\int_{0}^{L}\left(\frac{d}{d x}\left(\kappa p \frac{d p}{d x}\right)-q \frac{d}{d x}\left(\kappa \frac{d p}{d x}\right)\right) d x \\
& =\left(\kappa q \frac{d p}{d x}\right)_{0}^{L}-\int_{0}^{L} q \frac{d}{d x}\left(\kappa \frac{d p}{d x}\right) d x \\
\epsilon_{0} \int_{0}^{L} \frac{d \phi}{d x} \frac{d \psi}{d x} d x & =\epsilon_{0}\left(\phi \frac{d \psi}{d x}\right)_{0}^{L}+\int_{0}^{L} \phi\left(-\frac{d q}{d x}+\xi\right) \\
& =\zeta_{0} \phi_{0}+\zeta_{L} \phi_{L}+\int_{0}^{L}\left(\phi \xi+\frac{d \phi}{d x} q\right) d x
\end{aligned}
$$

where we have used Maxwell's to obtain the last equality. Putting all this together into Eq. (7),

$$
\begin{aligned}
& \int_{0}^{L}\left(-\frac{d}{d x}\left(\kappa \frac{d p}{d x}\right)+\frac{\partial W}{\partial p}(p, x)+\frac{d \phi}{d x}\right) q d x \\
& \quad+\int_{0}^{L}\left(\frac{\partial W_{\rho}}{\partial \rho}(\rho, x)+\phi\right) \xi d x++\left(\kappa \frac{d p}{d x} q\right)_{0}^{L}=0 .
\end{aligned}
$$

But this has to hold for arbitrary $q$, $\xi$; so we conclude that

$$
-\frac{d}{d x}\left(\kappa \frac{d p}{d x}\right)+\frac{\partial W}{\partial p}+\frac{d \phi}{d x}=0
$$




$$
\begin{gathered}
\frac{\partial W_{\rho}}{\partial \rho}+\phi=0, \\
\frac{d p}{d x}(0)=\frac{d p}{d x}(L)=0 .
\end{gathered}
$$

The first gives a relationship between the polarization and the electric field, the second a relationship between the charges and the electric potential, and the third is a natural boundary condition. These along with the Eqs. (2) and (3) are the governing differential equations for the variables $p$, $\rho$, and $\phi$. We shall assume that $W_{\rho}$ is convex, so that one can invert Eq. (15) uniquely to obtain $\rho=\rho(\phi)$, which we combine with the Maxwell Eq. (2).

In summary, the governing equations that determine the unknowns $p, \phi$ are

$$
\begin{gathered}
-\frac{d}{d x}\left(\kappa \frac{d p}{d x}\right)+\frac{\partial W}{\partial p}+\frac{d \phi}{d x}=0, \\
\frac{d}{d x}\left(-\epsilon_{0} \frac{d \phi}{d x}+p\right)=\rho(\phi),
\end{gathered}
$$

subject to the boundary conditions

$$
\phi(0)=\phi_{0}, \quad \phi(L)=\phi_{L}, \quad \frac{d p}{d x}(0)=0, \quad \frac{d p}{d x}(L)=0 .
$$

To close the system, we have to specify $\kappa, W$, and $\rho$. We shall assume that $\kappa$ is a fixed (material-dependent) constant for the rest of the paper. We shall assume that

$$
W(p)=\frac{a}{2}\left(T-T_{0}\right) p^{2}+\frac{b}{4} p^{4}+\frac{c}{6} p^{6},
$$

where $T$ is the absolute temperature, $a, c$, and $T_{0}$ are materials constants, and $b$ may be a constant or a function of $T$, depending on materials and the order of phase transition. For example, for barium titanate, $b=b_{0}\left(T-T_{1}\right)$, with $b_{0}$ and $T_{1}$ being constant. Finally, we assume that ${ }^{17}$

$$
\begin{aligned}
\rho(\phi, x)= & -e N_{c} F_{\frac{1}{2}}\left(\frac{E_{f m}-E_{c}+e \phi}{K_{B} T}\right) \\
& +e N_{\mu} F_{\frac{1}{2}}\left(\frac{E_{\mu}-e \phi-E_{f m}}{K_{B} T}\right) \\
& +z e N_{d}(x)\left(1-\frac{1}{1+\frac{1}{g} \exp \left(\frac{E_{d}-e \phi-E_{f m}}{K_{B} T}\right)}\right) \\
& -z^{\prime} e N_{a}(x)\left(1-\frac{1}{1+\frac{1}{g^{\prime}} \exp \left(\frac{E_{f m}+e \phi-E_{a}}{K_{B} T}\right)}\right)
\end{aligned}
$$

TABLE I. Material constants. ${ }^{1,18-20}$

\begin{tabular}{lccc}
\hline \hline Ferroelectric parameter & Properties units & $\mathrm{BaTiO}_{3}$ & $\mathrm{~Pb}\left(\mathrm{Zr}_{0.54} \mathrm{Ti}_{0.46}\right) \mathrm{O}_{3}$ \\
\hline$a$ & $\mathrm{JmC}^{-2} \mathrm{~K}^{-1}$ & $6.65 \times 10^{5}$ & $1.5 \times 10^{4}$ \\
$b$ & $\mathrm{Jm}^{5} \mathrm{C}^{-4}$ & $\ldots$ & $5.7 \times 10^{7}$ \\
$b_{0}$ & $\mathrm{Jm}^{5} \mathrm{C}^{-4} \mathrm{~K}^{-1}$ & $1.454 \times 10^{7}$ & $\ldots$ \\
$c$ & $\mathrm{Jm}^{9} \mathrm{C}^{-6}$ & $3.92 \times 10^{10}$ & $5.1 \times 10^{9}$ \\
$T_{0}$ & $\mathrm{~K}$ & 383 & \\
$T_{1}$ & $\mathrm{~K}$ & 448 & \\
$N_{c}$ & $\mathrm{~m}^{-3}$ & $10^{24}$ & $10^{24}$ \\
$N_{v}$ & $\mathrm{~m}^{-3}$ & $10^{24}$ & $10^{24}$ \\
$N_{d}$ & $\mathrm{~m}^{-3}$ & $10^{24} \sim 10^{26}$ & \\
$N_{a}$ & $\mathrm{~m}^{-3}$ & 0 & \\
$E_{c}$ & $\mathrm{eV}$ & -3.6 & 3.5 \\
$E_{v}$ & $\mathrm{eV}$ & -6.6 & 6.9 \\
$E_{d}$ & $\mathrm{eV}$ & -4.0 & \\
$E_{a}$ & $\mathrm{eV}$ & -6.2 & \\
\multicolumn{5}{c}{ Metal property } & $\left(E_{f m}\right.$ in eV & & \\
\multicolumn{5}{c}{ Platinum } & -5.3 & & \\
\multicolumn{5}{c}{ Bismuth } & -4.2 & & \\
\hline \hline
\end{tabular}

where $K_{B}$ is the Boltzmann constant, $e$ the Coulomb charge per electron, $N_{c}\left(N_{v}\right)$ the effective density of states in the conduction (valance) band, $E_{c}\left(E_{v}\right)$ the conduction (valance) band edge, $E_{d}\left(E_{a}\right)$ the donor (acceptor) level, $E_{f m}$ the Fermi level of the metal, $N_{d}\left(N_{a}\right)$ the donor (acceptor) density, $z\left(z^{\prime}\right)$ the valency of the donor (acceptor), $g\left(g^{\prime}\right)$ the ground state degeneracy of the donor (acceptor) level, and

$$
F_{\frac{1}{2}}(\eta)=\frac{1}{2 \sqrt{\pi}} \int_{0}^{\infty} \frac{\sqrt{\epsilon}}{1+\exp (\epsilon-\eta)} d \epsilon
$$

the Dirac-Fermi integral. It can easily be verified that, for each $x, \rho(\phi, x)$ is a monotone decreasing function, which justifies the convexity of $W_{\rho}$. In Table I, we list the material constants for barium titanate $\left(\mathrm{BaTiO}_{3}\right)$ and lead zirconate titanate (PZT) $;^{1,18-20}$ the work functions for common metals are also listed.

Before concluding this section, let us briefly comment on the stability of the solutions of Eqs. (14) and (15). A stable equilibrium solution, $p(x), \rho(x)$, is a local minimum of the potential energy $E$,

$$
\delta^{2} E=\left.\frac{d^{2}}{d \eta^{2}} E[p+\eta q, \rho+\eta \xi]\right|_{\eta=0} \geq 0
$$

for any $q(x), \xi(x)$. Evaluating this, we conclude that $p(x)$, $\rho(x)$ is a stable solution if

$$
\int_{0}^{L}\left\{\kappa\left(\frac{d q}{d x}\right)^{2}+q^{2} \frac{\partial^{2} W}{\partial p^{2}}(p(x), x)+\xi^{2} \frac{\partial^{2} W_{\rho}}{\partial \rho^{2}}(\rho(x), x)+\epsilon_{0}\left(\frac{d \psi}{d x}\right)^{2}\right\} d x \geq 0
$$


for any $q(x), \xi(x)$ where $\psi$ is obtained from $q$, $\xi$ as before as a solution to the Maxwell's Eq. (6). Since $\psi$ depends linearly on $q$ and $\xi$, according to Eq. (6), we can rewrite the integral above as a quadratic form in $q$ and $\xi$, involving the linearized operator. Thus, the condition above is exactly equivalent to the well-known condition of asymptotic stability, which requires the smallest eigenvalue of the linearized operator to be non-negative. ${ }^{21}$ Notice that, if $W, W_{\rho}$ are convex, then the solution is assuredly stable. The latter is true given our constitutive assumption on $\rho(\phi)$ in Eq. (18), but the former may fail for some solutions, since $W$ in Eq. (17) may have a multi-well structure at various temperatures. However, notice that the pointwise convexity of $W$ is not necessary, as the other terms may compensate for it in the integral stability condition of Eq. (21). For example, the integral may be positive, even if $p$ takes values in both the convex and concave domains of $W$, but if $\kappa$ is large enough. Therefore, we ignore stability issues when we study approximate solutions is Sec. III by taking $\kappa=0$. We note, however, that the numerical method that we use in Sec. IV automatically picks a stable equilibrium solution.

\section{COMPUTATIONAL RESULTS}

We solve the governing Eqs. (14) and (15) subject to Eq. (16) using collocation method. Since these equations may have multiple solutions, the numerical solution depends on the initial guess. The method ensures that we only obtained stable solutions (local minima). We present our results for homogeneous n-type $\mathrm{BaTiO}_{3}$ films with Pt electrodes at $300 \mathrm{~K}$. Therefore, $N_{a}=0$ in Eq. (18).

We begin with zero applied voltage or short-circuited films. The series of three figures on the left column of Fig. 1 show the results for three different thicknesses with dopant (donor) concentration of $5.0 \times 10^{24} \mathrm{~m}^{-3}$. When the film is very thin (e.g., $10 \mathrm{~nm})$, the polarization is linear near the saturation value of $-0.27 \mathrm{C} / \mathrm{m}^{2}$ and the potential slightly bends upward. As the film thickness increases, the polarization continues to be linear with almost the same slope and the potential rises further until, at around $200 \mathrm{~nm}$, we begin to see a distinct change. The polarization and the potential tend to become constant near the middle of the film. The middle region continues to grow with increasing film thickness, and we obtain a film with almost constant polarization and potential with distinct boundary layers, as shown for $1000 \mathrm{~nm}$. Figure 2 shows the space charges in these films. As we can see, the film is fully depleted for thin films, as was assumed in the work of Baudry and Tournier, ${ }^{22}$ but only the boundary layers are depleted in the thick films. We can indeed estimate the width of the depletion layer $(d)$ using the approximate model in Sec. IV: ${ }^{15,29} d \approx \sqrt{2 \epsilon_{r} \epsilon_{0} \phi_{c} / e z N_{d}}$, where $\epsilon_{r}=\left(\left.\frac{\partial^{2} W}{\partial p^{2}}\right|_{p 0} \epsilon_{0}\right)^{-1}$. Finally, we note that we can have another solution near the positive saturation polarization at each of these film thicknesses. Thus, films at this dopant concentration behave like classical undoped films.

The situation is dramatically different as the dopant concentration is increased beyond a critical value. The series of four figures on the left column of Fig. 3 show the results for four different thicknesses with a higher dopant (donor) con-
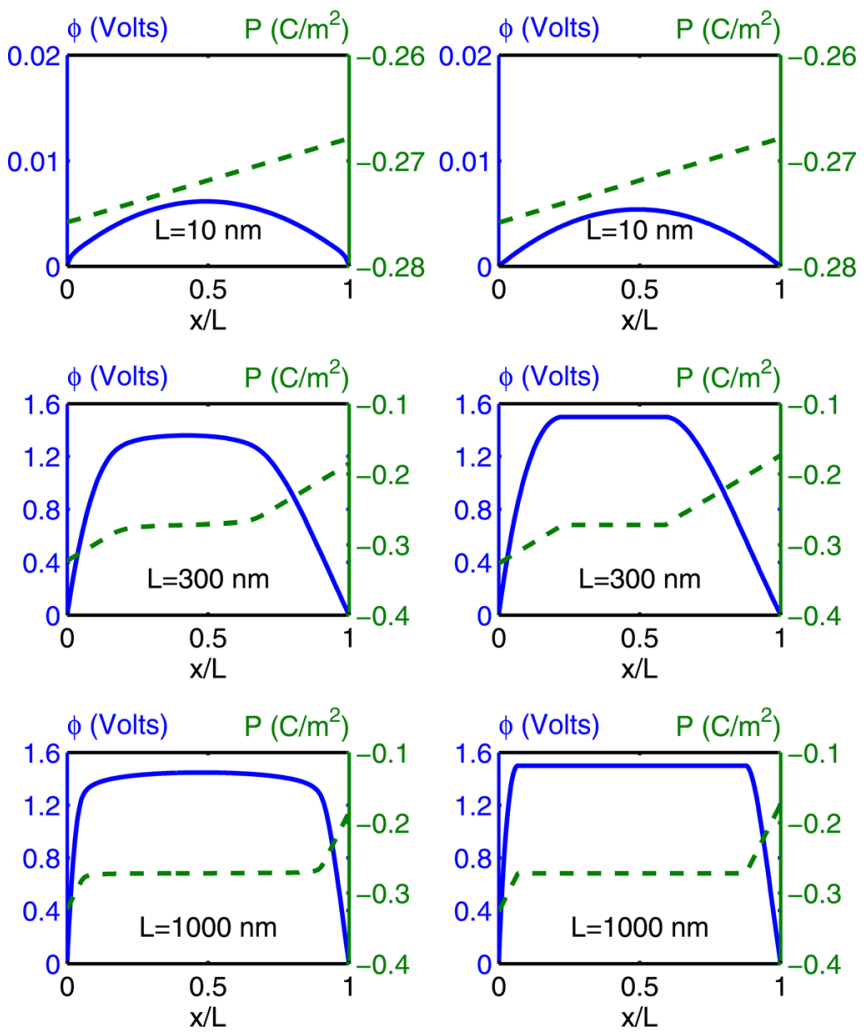

FIG. 1. Electric potential and polarization profiles in n-type $\mathrm{BaTiO}_{3}$ films at $300 \mathrm{~K}$ with dopant density of $5.0 \times 10^{24} \mathrm{~m}^{-3}$ for various thicknesses. The left column shows the computational results of the full model, while the right column shows the results of the approximate model. The solid blue lines are potential, and the dashed green lines are the polarization.

centration of $5.0 \times 10^{25} \mathrm{~m}^{-3}$. We do not see much difference when the film is very thin (e.g., $10 \mathrm{~nm}$ ): the films are fully depleted and the polarization is linear around the saturation value of $-0.27 \mathrm{C} / \mathrm{m}^{2}$. Also, as before, there is another solution near the positive saturation polarization. However, dramatic differences begin to emerge as the thickness increases. The polarization profile is still linear, but explores a large range starting from positive to negative, as shown for $60 \mathrm{~nm}$ and $100 \mathrm{~nm}$. Further, the potential profile eventually develops two

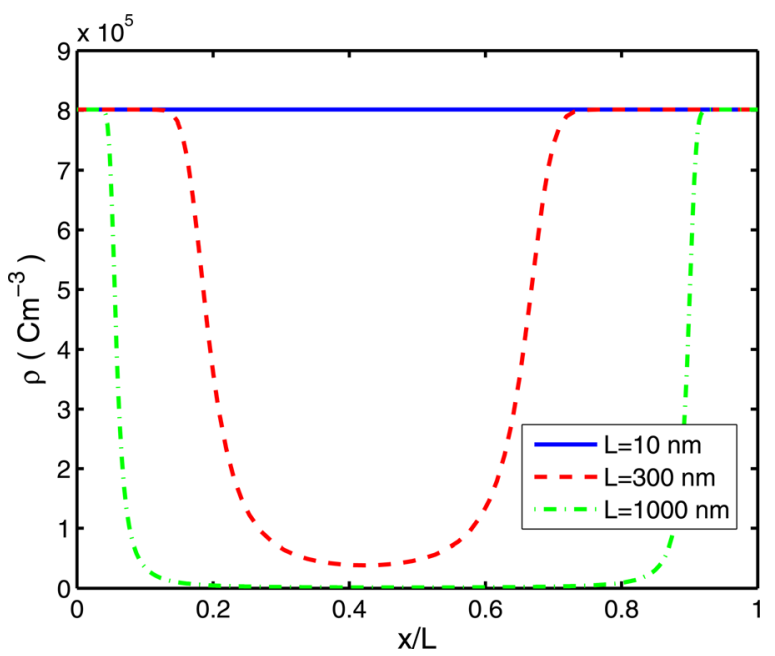

FIG. 2. Space charges in n-type $\mathrm{BaTiO}_{3}$ films at $300 \mathrm{~K}$ with dopant density of $5.0 \times 10^{24} \mathrm{~m}^{-3}$ for various thicknesses. 

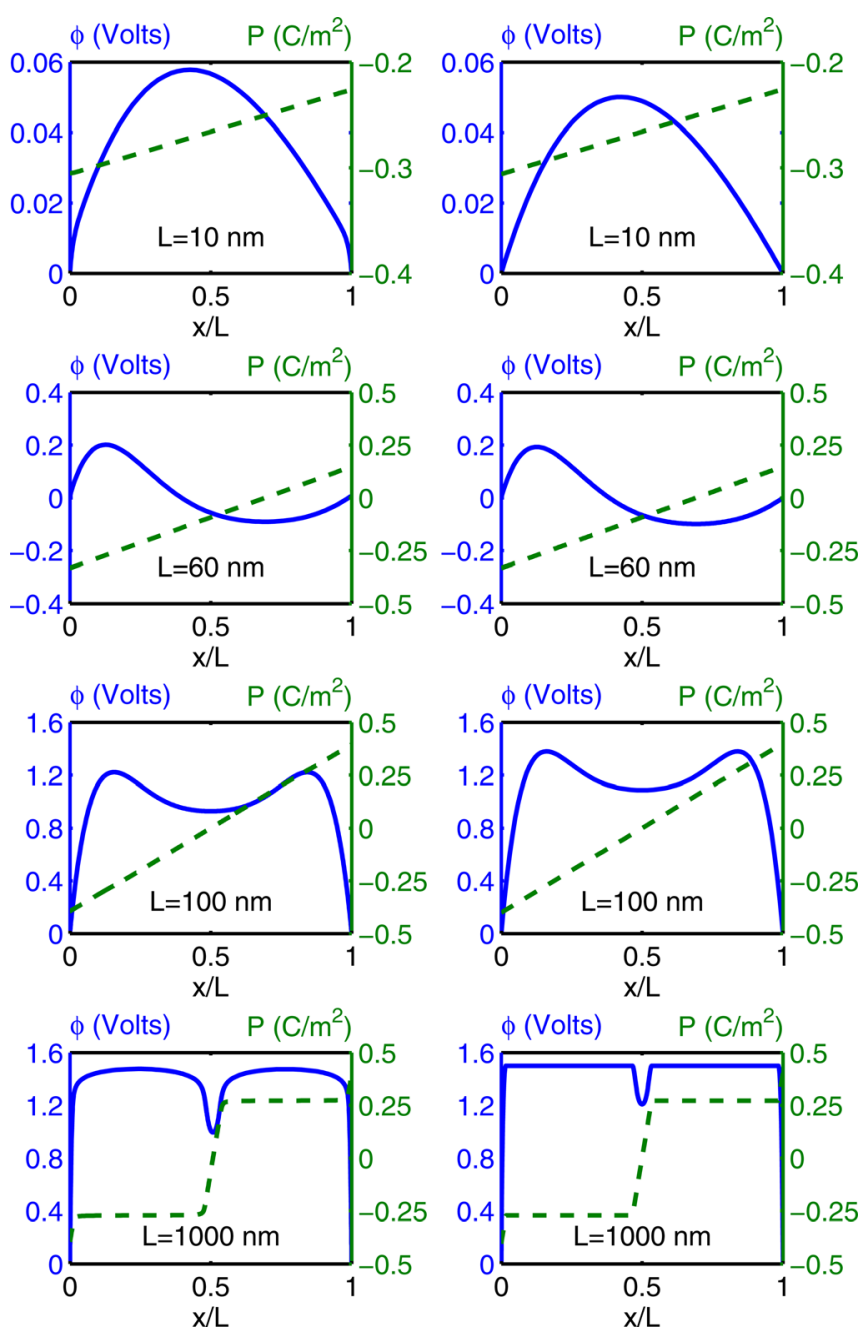

FIG. 3. Electric potential and polarization profiles in n-type $\mathrm{BaTiO}_{3}$ films at $300 \mathrm{~K}$ with dopant density of $5.0 \times 10^{25} \mathrm{~m}^{-3}$ for various thicknesses. The left column shows the computational results of the full model, while the right column shows the results of the approximate model. The solid blue lines are potential, and the dashed green lines are the polarization.

humps (e.g., $100 \mathrm{~nm}$ ). Furthermore, there is only one solution, suggesting a loss of switching behavior. As the film thickness increases further, the polarization has a tail-to-tail profile, with one region constant at the positive saturation value and another at the negative saturation value separated by a transition layer and with two boundary layers at the two ends (e.g., $1000 \mathrm{~nm}$ ). There is, in fact, a one-parameter family of solutions (the position of the central transition layer is indeterminate); the one shown here is just one of them, chosen to have a symmetric potential profile. This suggests the complete shielding of the polarization by space charges. All of this is emphasized in Fig. 4, which shows the space charge distribution for various thicknesses. The film is fully depleted at very thin thicknesses (e.g., $10 \mathrm{~nm}$ and $60 \mathrm{~nm}$ ). At larger thickness, the film has three depletion layers - two boundary layers and one transition layer in the middle. This suggests the loss of ferroelectricity in intermediate and thick films of highly doped material.

We now examine the switching behavior of films with various thicknesses and dopant concentrations by studying their switching fields and hysteresis loops. Since the polarization

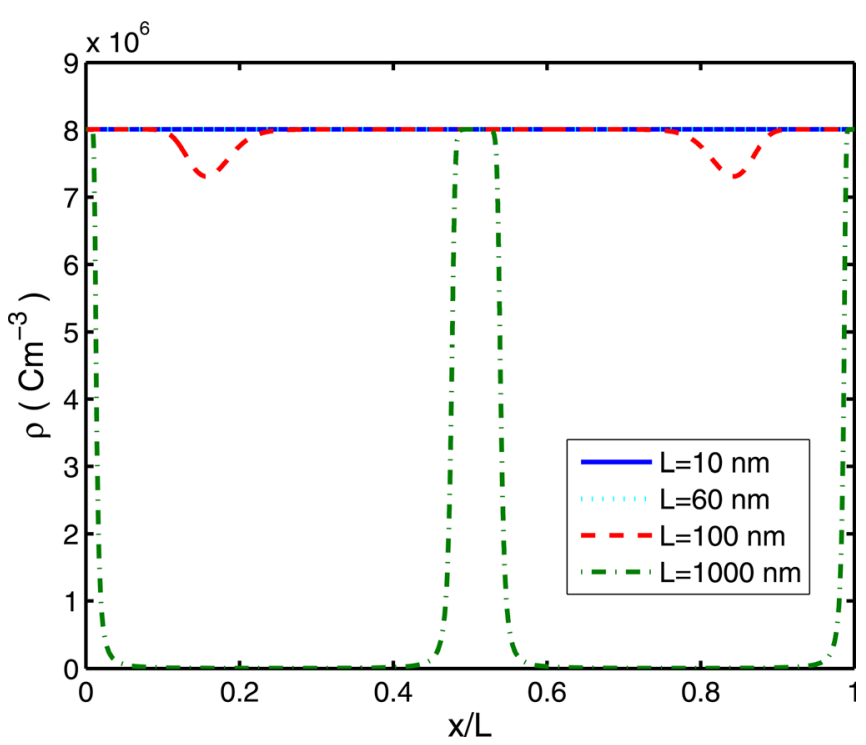

FIG. 4. Space charges in n-type $\mathrm{BaTiO}_{3}$ films at $300 \mathrm{~K}$ with dopant density of $5.0 \times 10^{25} \mathrm{~m}^{-3}$ for various thicknesses.

across the films is not uniform, we use the effective polarization based on the charges induced on the top plate at $x=L$ in plotting the hysteresis loops. This is a reasonable choice, considering the common Sawyer-Tower circuit used in observing the ferroelectric hysteresis loops. ${ }^{18}$ In this setup, a linear capacitor $C_{0}$ with known capacitance is connected in series with the specimen film. The $\mathrm{Y}$ signal in the hysteresis loop is indeed the voltage across $C_{0}$ or, equivalently, the charges induced on $C_{0}$, which is assumed to be equal to the charges induced on the specimen film (i.e., the electric displacement $D$ on the surface), and thus approximates the polarization of the film, the film is ferroelectric and $p \gg \epsilon_{0} E$. We shall return to discuss the consequences of this choice in some detail later.

We begin with a low dopant concentration of $5.0 \times 10^{24} \mathrm{~m}^{-3}$. Figure 5 shows the effective polarization as a function of applied electric field for thick films of $1000 \mathrm{~nm}$. In doing these calculations, we gradually increase or decrease the applied field using the previous state as the initial guess. $E_{c}$ in the abscissa is the slope of the DevonshireLandau energy at the spinodal point (Fig. 15),

$$
E_{c}=\left|\frac{\partial W}{\partial p}\left( \pm p_{s}\right)\right| \quad \text { where } \quad \frac{\partial^{2} W}{\partial^{2} p}\left( \pm p_{s}\right)=0
$$

or the classical coercive field. For the material parameters we choose for $\mathrm{BaTiO}_{3}$ in Table I at $300 \mathrm{~K}, E_{c}=1.572$ $\times 10^{7} \mathrm{Vm}^{-1}$. We notice that the switching field $E_{s}$ in Fig. 5 is significantly smaller than the classical value $E_{c}$. The insets in the figure show the polarization and potential profiles at four different points. Starting from (A), where the film is under positive electric field, the polarization near the electrode at $x=L$ gradually marches to the negative domain (B) with increasing negative electric field, while the majority of the polarization profile across the film is kept unchanged. Upon reaching the switching field, the polarization profile across the whole film suddenly changes to the negative domain $(\mathrm{C})$. The switching process from (C) to (D) to (A) is similar-only a small portion of the film is involved most of 
the time. This implies that the boundary layers near the electrodes provide a mechanism for the nucleation of an opposite-polarized region, which enables switching at a lower field than $E_{c}$. Recall, however, that the boundary layers disappear at smaller thicknesses at this doping layer. We find a higher switching field, as shown in Fig. 6, for a 100-nm film.

In addition, we notice that the hysteresis loop for the $1000-\mathrm{nm}$ case is asymmetric in the sense that the switching field under positive field is smaller than the one under negative field. This asymmetry has been widely observed in both bulk ferroelectrics (e.g., Arlt and Neumann ${ }^{23}$ in Ni-doped $\mathrm{BaTiO}_{3}$, Hall and Ben-Omran ${ }^{24}$ in Co-doped $\mathrm{BaTiO}_{3}$ ), where it has been described as the development of an internal bias field, as well as in thin films (e.g., Warren et al. ${ }^{25}$ and Okamora et al. ${ }^{26}$ in Pt/PZT/Pt films). For the 100-nm case, the hysteresis loop is more symmetric, as obviously shown in Fig. 6. We elaborate on this asymmetry in Fig. 7. The blue stars are for positive electric field, while the red circles are for negative electric field. As obvious from the figure, the switching field increases with decreasing film thickness and
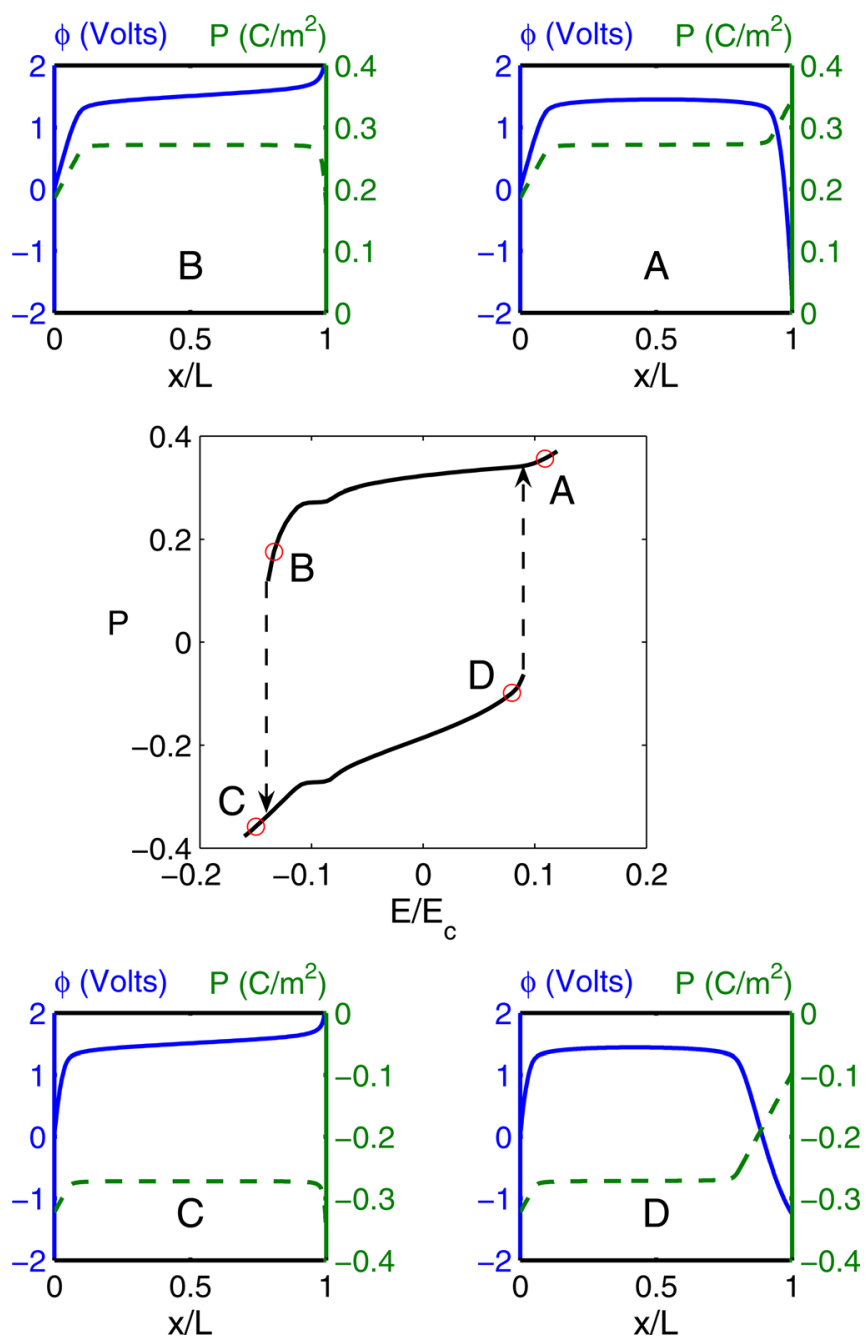

FIG. 5. The effective polarization vs electric field hysteresis curves for a 1000-nm n-type $\mathrm{BaTiO}_{3}$ film at $300 \mathrm{~K}$ with dopant density of $5.0 \times 10^{24} \mathrm{~m}^{-3}$. The insets show the polarization and potential profiles at the points $A, B, C$, and $D$.
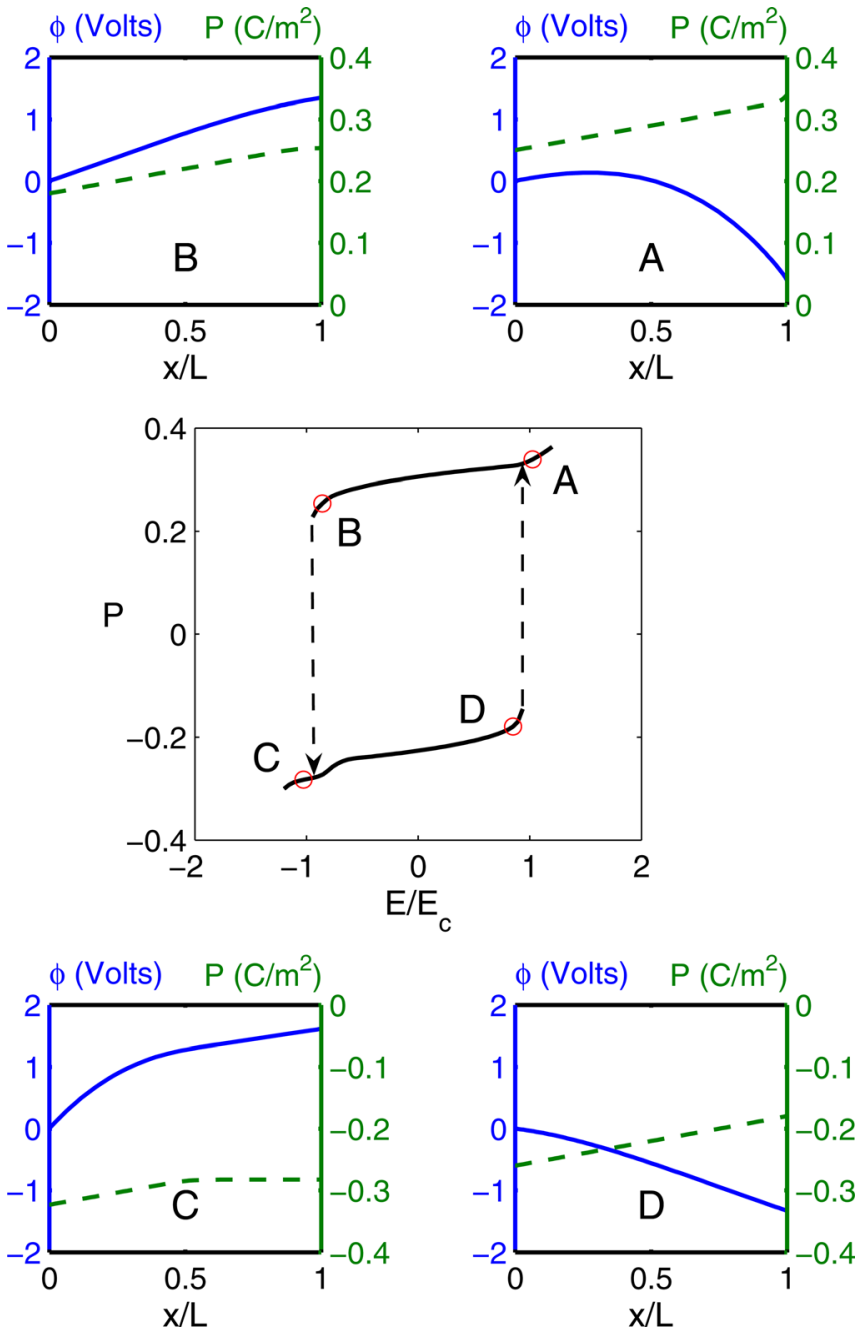

FIG. 6. The effective polarization vs electric field hysteresis curves for a 100 -nm n-type $\mathrm{BaTiO}_{3}$ film at $300 \mathrm{~K}$ with dopant density of $5.0 \times 10^{24} \mathrm{~m}^{-3}$. The insets show the polarization and potential profiles at the points $A, B, C$, and $D$.

reaches the classical value in the limit of zero thickness. In addition, the switching field is symmetric only in very thin films. For thicker films, the switching field under positive field is substantially lower than the one under negative field.

Even though the Pt-BaTiO 3 -Pt configuration is symmetric, there are, in fact, two sources of asymmetry in the situation under consideration. The first source is the fact that we consider materials that are assumed to be n-type doped. This causes the switching field under positive field to be lower than the one under negative field. The second is that we are reporting this polarization based on the charge at the top plate $(x=L)$. For a classical ferroelectric film with $\rho=0$, from the Maxwell Eq. (2), the electric displacement $D$ is constant across the film. However, for a doped film, these quantities are no longer constants across the film. In particular,

$$
D_{x=L}=D_{x=0}+\int_{0}^{L} \rho d x=D_{x=0}+Q,
$$

where $Q$ is the space charges stored in the film. Thus, one would measure a different response if the reference capacitor 

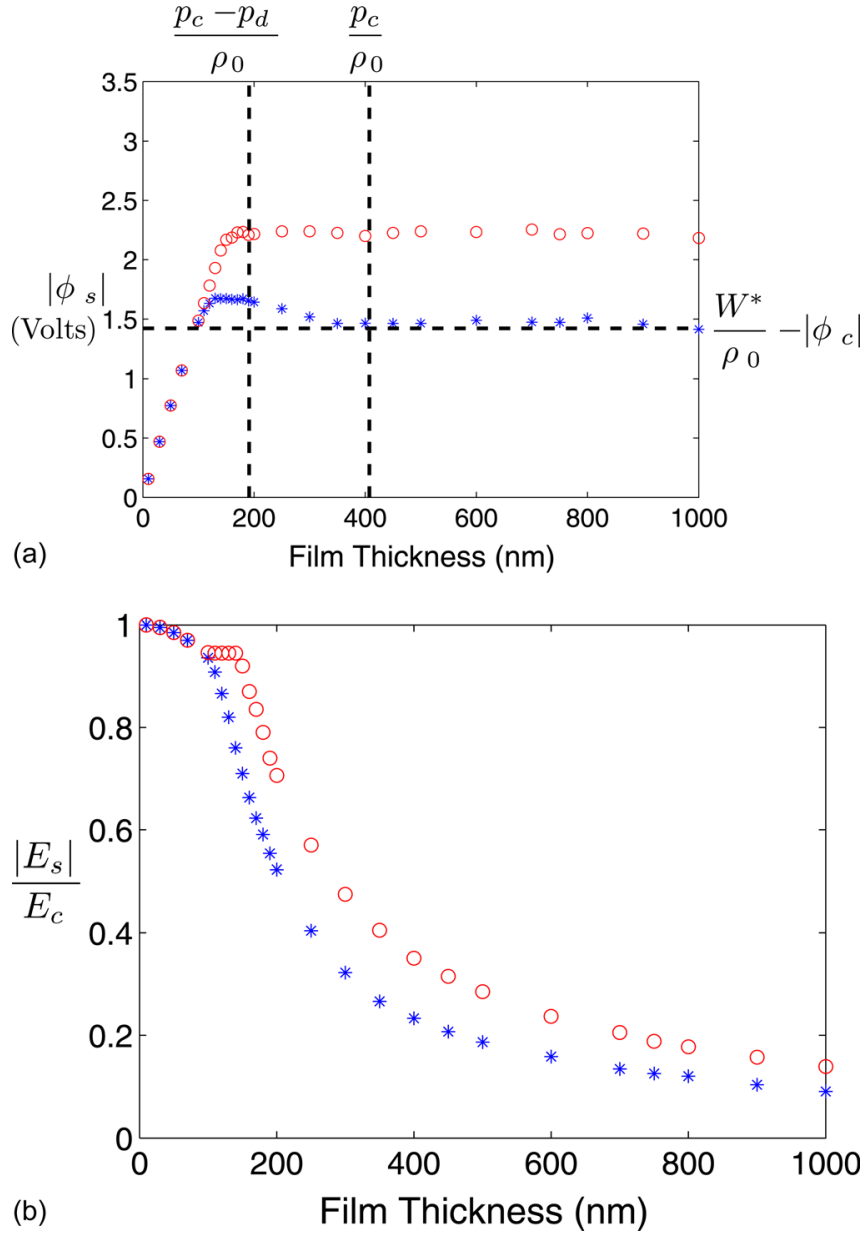

FIG. 7. The potential (a) and field (b) required for switching a film with low doping $\left(N_{d}=5.0 \times 10^{24} \mathrm{~m}^{-3}\right)$. The red circles are for negative electric field, while the blue stars are for positive electric field.

were connected to the bottom plate (e.g., Zheng et al. ${ }^{27}$ ) These values also differ from the spatial average of the polarization, which is commonly reported in computational studies: this tends to be more symmetric, since it samples the bulk and is relatively unaffected by the depletion layers.

Another interesting finding of Fig. 7 is that the switching potential is almost constant beyond some critical film thickness. We will revisit this in Sec. IV.

At the high dopant concentration of $5.0 \times 10^{25} \mathrm{~m}^{-3}$, very thin films behave similarly as the low doping case, as shown in Fig. 8. However, for thicker films, the switching behavior is quite different, as demonstrated by Figs. 9 and 10 , for a high-doped film with thickness of $100 \mathrm{~nm}$ and $500 \mathrm{~nm}$, respectively. The hysteresis loops now reside entirely in the negative field domain. In addition, in both figures, by looking into the polarization profiles of points A, B, $\mathrm{C}$, and $\mathrm{D}$, we find that the polarization at $x=0$ is almost fixed, regardless of the applied field. Recall from Fig. 3 that, for these thicknesses, the emergence of a central dip in the voltage profile suggests a loss of traditional ferroelectricity. Thus, these unusual hysteresis loops may reflect space charge leakage instead of polarization.

To explore this, we recall that the hysteresis loops are plotted using the polarization at $x=L$. In Figs. 11, 12, and 13, we show the result of alternate choices. From these three
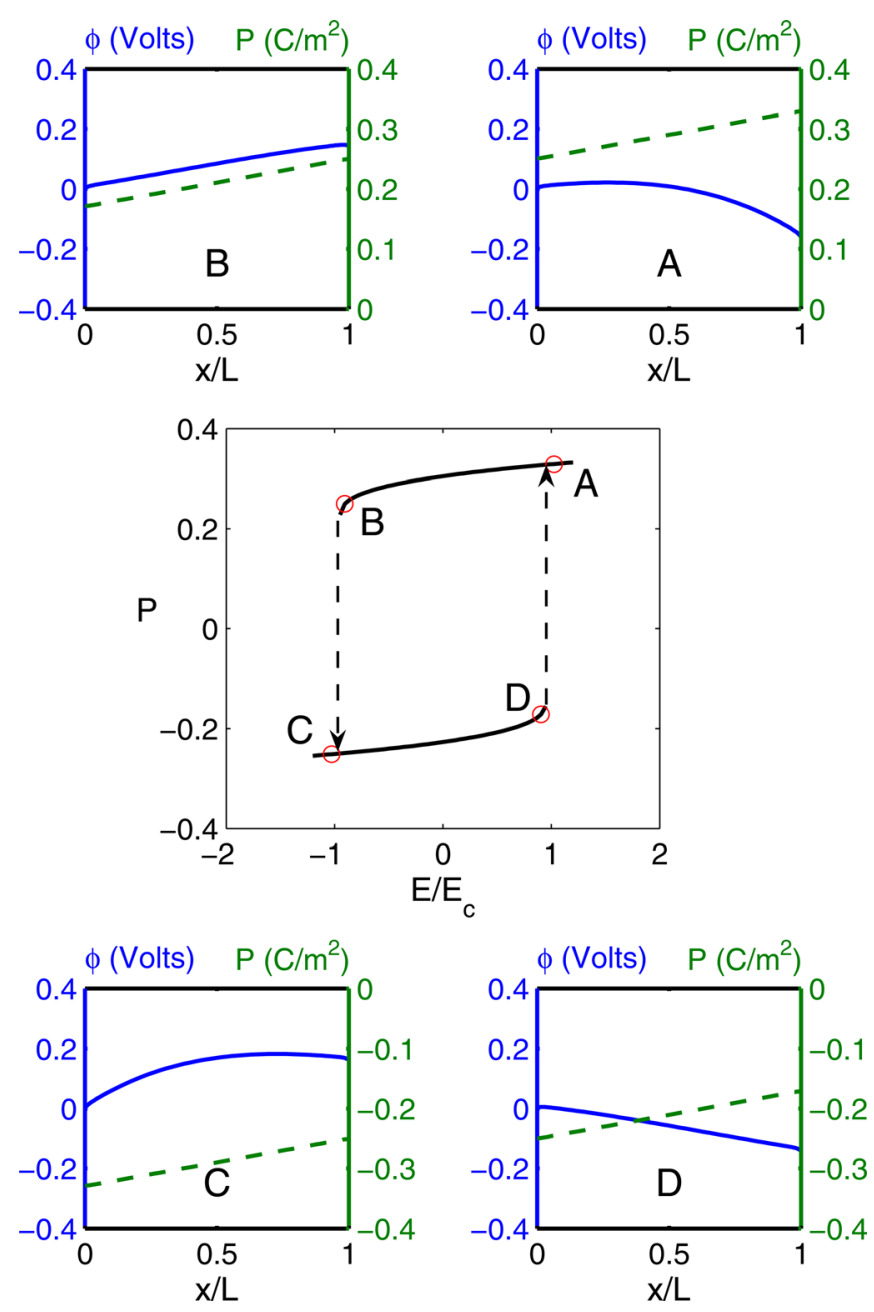

FIG. 8. The effective polarization vs electric field hysteresis curves for a 10 -nm n-type $\mathrm{BaTiO}_{3}$ film at $300 \mathrm{~K}$ with dopant density of $5.0 \times 10^{25} \mathrm{~m}^{-3}$. The insets show the polarization and potential profiles at the points $A, B, C$, and $D$.

figures, we can see that the polarization $p$ still approximates the electric displacement $D$ at the two ends: The black line ( $p$ at $x=L$ ) is covered by blue crosses $(D$ at $x=L$ ), which, in turn, are covered by green diamonds (sum of $Q$ and $D$ at $x=0$ ), and the magenta line ( $p$ at $x=0$ ) is covered by yellow crosses $(D$ at $x=0)$.

For a $10-\mathrm{nm} \mathrm{BaTiO}_{3}$ film with high dopant density, as shown in Fig. 11, we notice that the total charge $Q$ stored in the film is almost constant, with a positive value under different electric field. Therefore, the hysteresis loops measured by charges at the top electrode and bottom electrode are a simple vertical shift from each other. The difference is related to the contribution to the polarization by the space charges. This is consistent with the observations of Dawber et al., ${ }^{28}$ who found that extrapolating the measured inverse capacitance versus thickness in lead-zirconate-titanate (PZT) films to zero thickness gives a nonzero intercept.

In contrast, for a $100-\mathrm{nm}$ and a $500-\mathrm{nm} \mathrm{BaTiO}_{3}$ film with high dopant density, as shown in Fig. 12 and Figure 13, respectively, the polarization measured at the top and the bottom electrodes are completely different: the bottom electrode shows no apparent polarization, while the top electrode does. We also notice that the difference is consistent with 

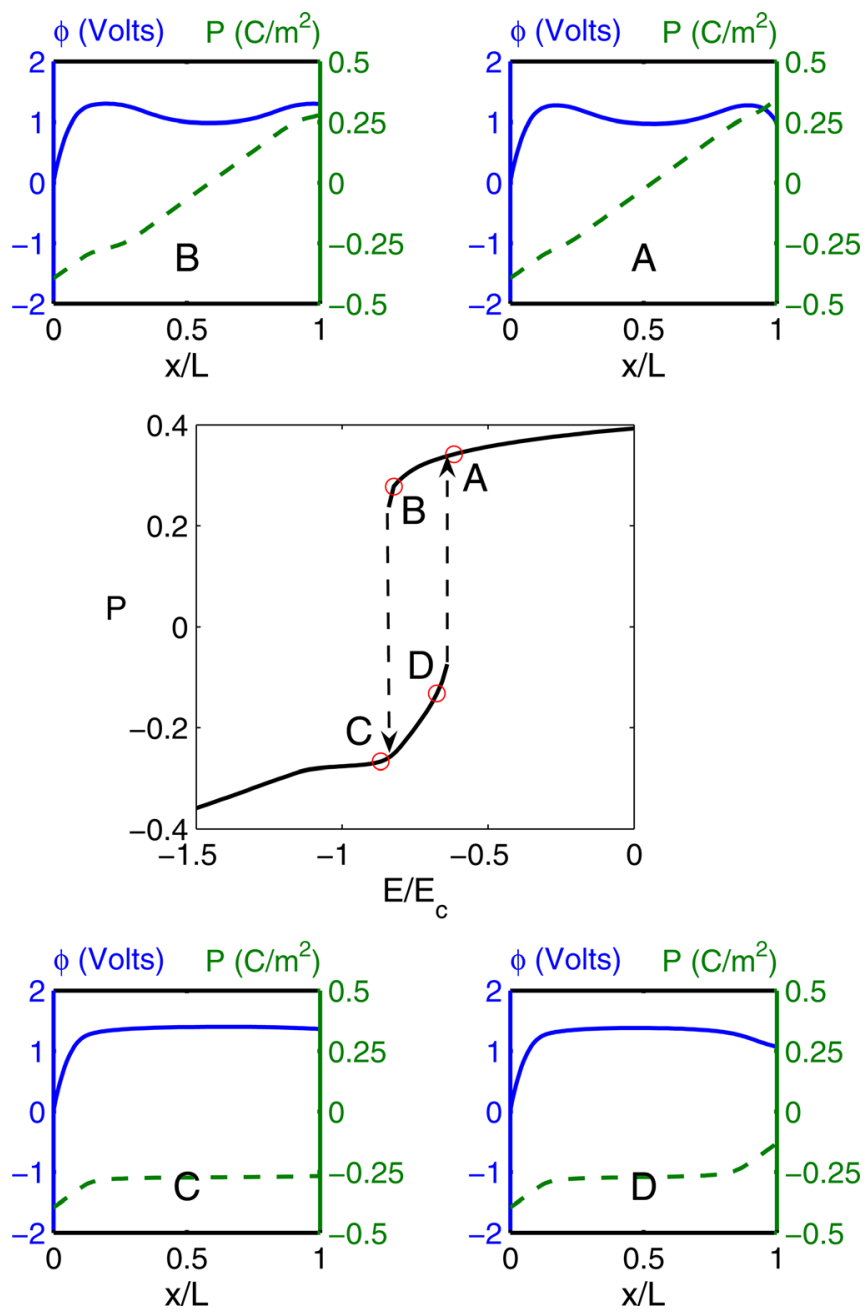

FIG. 9. The effective polarization vs electric field hysteresis curves for a 100 -nm n-type $\mathrm{BaTiO}_{3}$ film at $300 \mathrm{~K}$ with dopant density of $5.0 \times 10^{25} \mathrm{~m}^{-3}$. The insets show the polarization and potential profiles at the points $A, B, C$, and $D$.

change in total space charge $Q$ through the cycle. In other words, these films are not ferroelectric, confirming our earlier speculation.

In summary, our numerical simulations show that the films with low dopant concentration behave like classical films, except for depletion layers at larger thickness, which enables easy switching. For films with high dopant concentration, the classical behavior is preserved only by very thin films. For thicker films, a significant part of the film remains unswitched, no matter how high the applied field is. The hysteresis loop is more a reflection of the space charges than the true polarization status of the film. The superficially high polarization at $x=L$ induced by spaces charges is highly unsustainable, because of leakage (the so-called retention problem). ${ }^{1}$ Therefore, we conclude that thick films with high dopant concentration are not suitable for conventional applications, such as in memory devices or actuators.

\section{ANALYSIS OF AN APPROXIMATE MODEL}

To understand the behavior in some detail, we simplify our model. The highest (second) order terms in the governing Eqs. (14) and (15) are linear, with very small coefficients $\kappa$
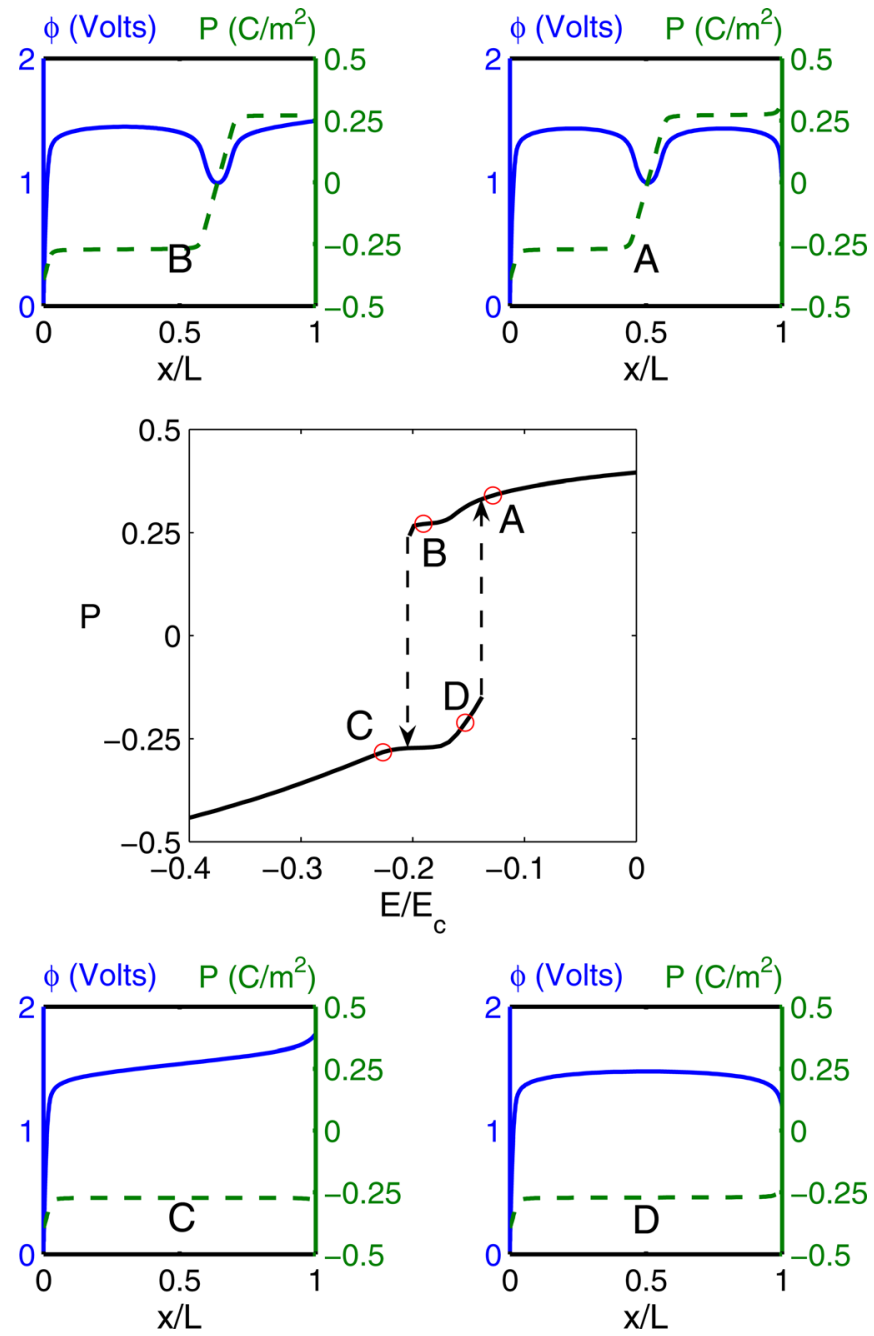

FIG. 10. The effective polarization vs electric field hysteresis curves for a 500 -nm n-type $\mathrm{BaTiO}_{3}$ film at $300 \mathrm{~K}$ with dopant density of $5.0 \times 10^{25} \mathrm{~m}^{-3}$. The insets show the polarization and potential profiles at the points $A, B, C$, and $D$.

and $\epsilon_{0}$. Thus, the behavior is driven by the nonlinear lower terms, with the second order terms smoothing things out in boundary and transition layers. Thus, we can understand the essential behavior by dropping the second order terms in Eqs. (14) and (15), i.e., by assuming that $\kappa=\epsilon_{0}=0$. Thus, we consider

$$
\begin{gathered}
\frac{d W}{d p}+\frac{d \phi}{d x}=0, \\
\frac{d p}{d x}=\rho(\phi),
\end{gathered}
$$

subject to the boundary conditions

$$
\phi(0)=\phi_{0}, \quad \phi(L)=\phi_{L}
$$

We further assume, by examining Eq. (18) and Table I that

$$
\rho(\phi)=\left\{\begin{array}{cc}
0 & \phi \geq \phi_{c}, \\
\rho_{0} & \phi<\phi_{c}
\end{array}\right.
$$




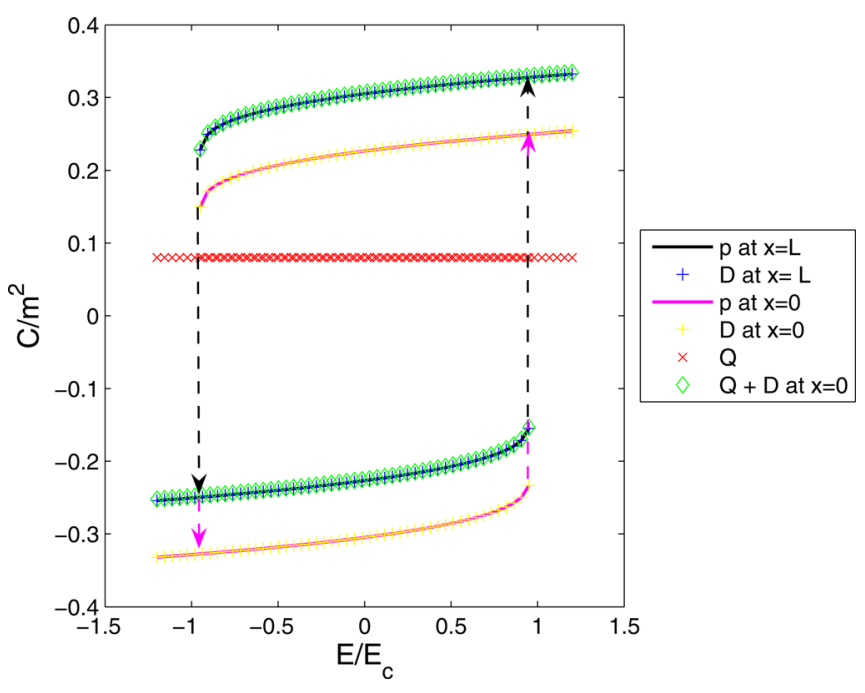

FIG. 11. The hysteresis loops formed by various quantities for a 10-nm n-type $\mathrm{BaTiO}_{3}$ film at $300 \mathrm{~K}$ with dopant density of $5.0 \times 10^{25} \mathrm{~m}^{-3}$.

where $\phi_{c}$ is the ideal build-in potential for a semiconducting film coated with metal electrodes. For the n-type $\mathrm{BaTiO}_{3}$ film and platinum electrodes at $300 \mathrm{~K}$, we consider, in this paper, $\phi_{c}=\left(E_{f m}^{B T}-E_{f m}^{P t}\right) / e=\left(E_{c}+E_{d}-2 E_{f m}^{P t}\right) / 2 e=1.5 \mathrm{~V}$. And $\rho_{0}=e N_{d}>0$, assuming the valency of the donors is 1 . Please refer to $\mathrm{Xiao}^{29}$ for detailed discussion of this simplification. We can now integrate these equations explicitly by dividing them into three regimes.
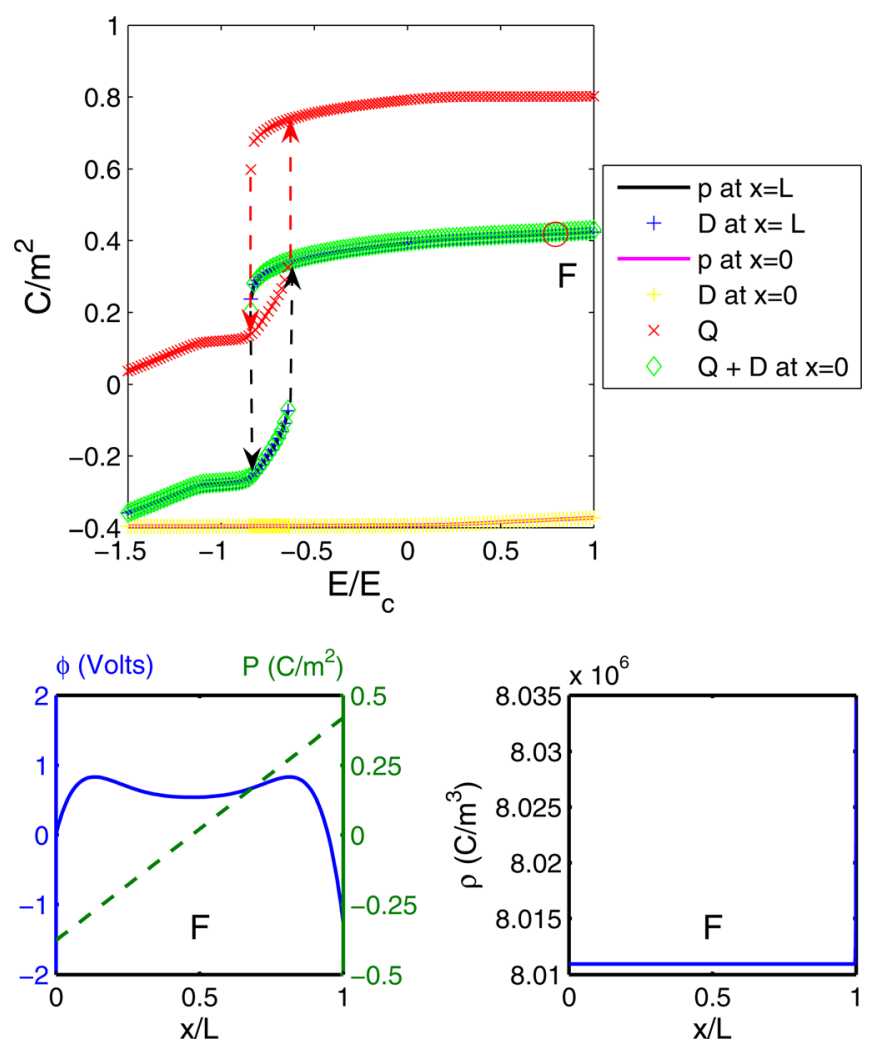

FIG. 12. The hysteresis loops formed by various quantities for a $100-\mathrm{nm}$ n-type $\mathrm{BaTiO}_{3}$ film at $300 \mathrm{~K}$ with dopant density of $5.0 \times 10^{25} \mathrm{~m}^{-3}$. The insets show the polarization, potential, and space charge profiles at point $\mathrm{F}$.
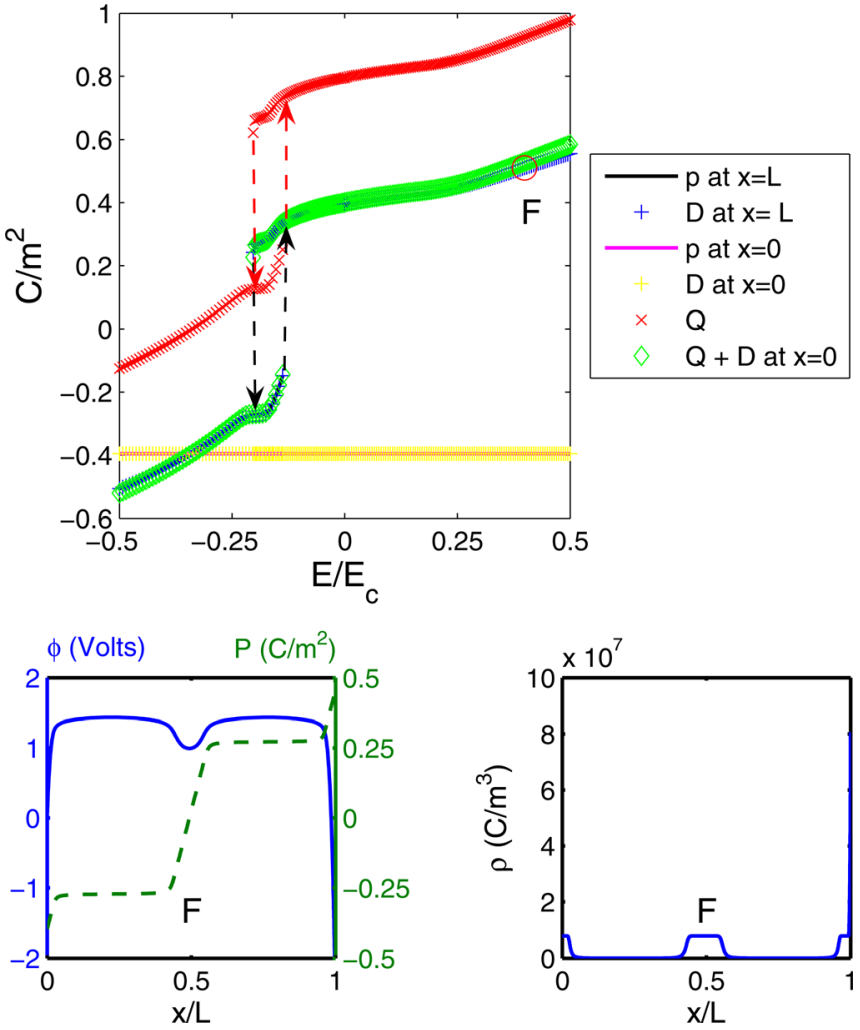

FIG. 13. The hysteresis loops formed by various quantities for a $500-\mathrm{nm}$ n-type $\mathrm{BaTiO}_{3}$ film at $300 \mathrm{~K}$ with dopant density of $5.0 \times 10^{25} \mathrm{~m}^{-3}$. The insets show the polarization, potential, and space charge profiles at point $\mathrm{F}$.

Regime I. Assume $\phi<\phi_{\mathrm{c}}$ on some interval $\left(x_{1}, x_{2}\right)$. Then, $\rho=\rho_{0}$, according to Eq. (27). We conclude, from Eq. (25), that

$$
p(x)=\rho_{0} x+k
$$

for some constant $k$. It follows, then, from Eq. (24) that

$$
\begin{aligned}
\phi(x) & =\int-\frac{d W}{d p}\left(\rho_{0} x+k\right) d x+\hat{k} \\
& =\int-\frac{d W}{d p}(p)\left(\frac{d p}{d x}\right)^{-1} d p+\hat{k}=-\frac{1}{\rho_{0}} W\left(\rho_{0} x+k\right)+\hat{k}
\end{aligned}
$$

for some constant $\hat{k}$. Thus, in regime $\mathrm{I}$, the polarization is linear with slope $\rho_{0}$, while the potential $\phi$ follows the graph of $-W$ scaled vertically and horizontally by $\rho_{0}$.

Regime II. Assume $\phi \equiv \phi_{c}$ on some interval $\left(x_{1}, x_{2}\right)$. Then, $\rho=0$, and we conclude from Eq. (25) that

$$
p(x)=k=\text { constant }
$$

It follows, then, from Eq. (24), that

$$
\frac{d W}{d p}(k)=0 .
$$

Thus, in regime II, the polarization $p$ is constant at an equilibrium point of $W$, and the potential $\phi$ is constant at $\phi_{c}$. 
Regime III. Assume $\phi>\phi_{c}$ on some interval $\left(x_{1}, x_{2}\right)$. Then, $\rho=0$, and we conclude from Eq. (25) that

$$
p(x)=k=\text { constant }
$$

It follows, then, from Eq. (24), that

$$
\phi(x)=-\frac{d W}{d p}(k) x+\hat{k}
$$

for some constant $\hat{k}$. Thus, in regime III, the polarization $p$ is constant, and the potential $\phi$ is linear with slope $-d W / d p$.

We can now construct solutions by piecing together solutions from different regimes. Since $\phi$ follows the graph of $-W$ scaled vertically and horizontally by $\rho_{0}$ when $\phi<\phi_{c}$, the doping level and the film thickness are two very important parameters in constructing the solutions. We demonstrate this for a film at a temperature (e.g., $300 \mathrm{~K}$ considered in this paper) below the Curie temperature so that $W$ has two minima at $\pm p_{0}$ and a maximum at 0 . Let $W^{*}=\left|W\left(p_{0}\right)\right|=-W\left(p_{0}\right)$ be the energy barrier between the two minima and $\pm p^{*} \neq 0$ be the points where $W\left( \pm p^{*}\right)=0$, as shown in Fig. 14. It is convenient to consider two cases separately.

- Low doping: $\rho_{0}\left|\phi_{c}\right|<W^{*}$

- High doping: $\rho_{0}\left|\phi_{c}\right|>W^{*}$

For the material parameters we choose for $\mathrm{BaTiO}_{3}$ in Table I at $300 \mathrm{~K}, W^{*}=2.34 \times 10^{6} \mathrm{Jm}^{-3}$, and the critical doping level that differentiates the high/low doping cases is $9.74 \times 10^{24} \mathrm{~m}^{-3}$.
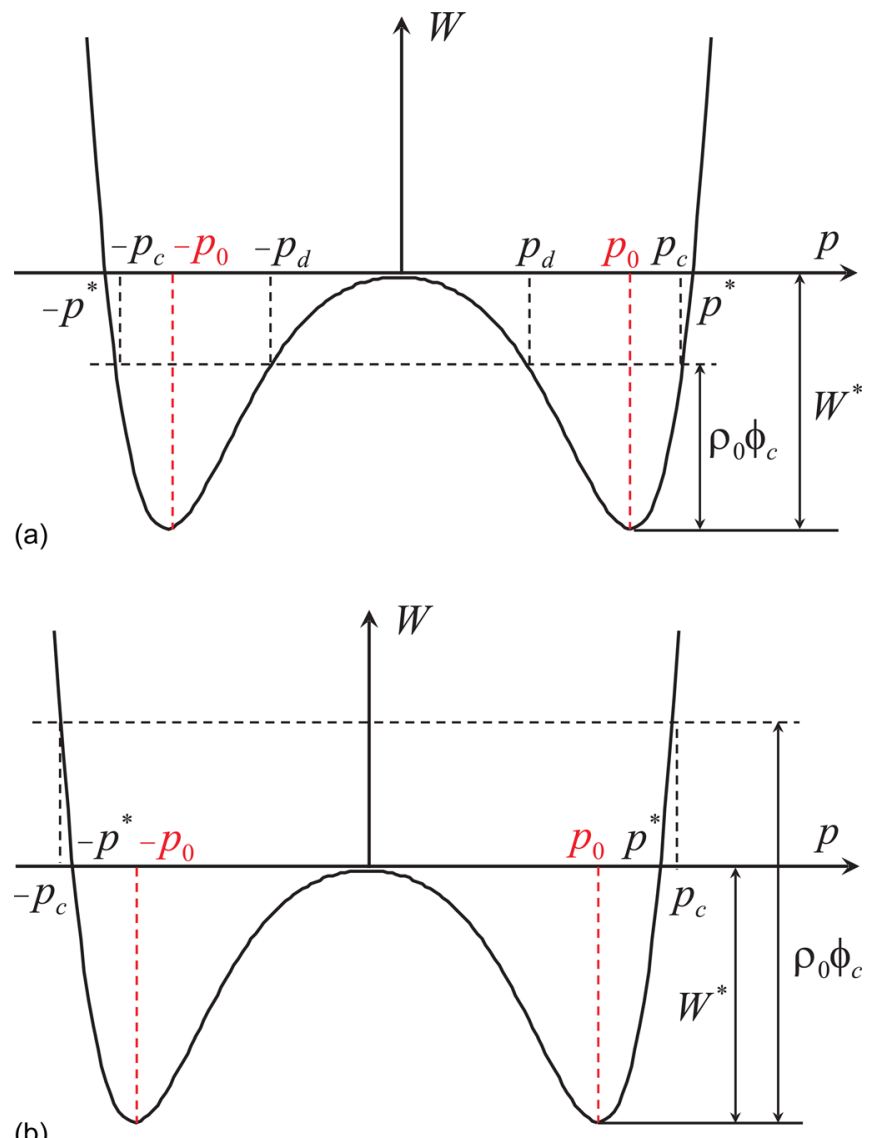

(b)

FIG. 14. The Devonshire-Landau energy and terminology for low doping (a) and high doping (b).

\section{A. Low doping}

We assume that $\rho_{0}\left|\phi_{c}\right|<W^{*}$ in this section. Let $\pm p_{d}$ and $\pm p_{c}\left(0<p_{d}<p_{0}<p_{c}\right)$ be the points, as shown in Fig. 14(a), which satisfy

$$
W\left( \pm p_{d}\right)=W\left( \pm p_{c}\right)=W\left(p_{0}\right)+\rho_{0}\left|\phi_{c}\right| .
$$

We can have three types of films depending on thickness. For the material parameters and doping level we consider in Fig. 1, films with $L<191 \mathrm{~nm}$ belong to the thin film regime, films with $L>815 \mathrm{~nm}$ belong to the thick film regime, and the intermediate film regime incudes films with thickness in between.

Thin films. Let $\rho_{0} L<p_{c}-p_{d}$. We begin with the case of zero applied field, $\phi_{0}=\phi_{L}=0$. It is possible to find solutions which only use regime I. We use the boundary conditions to find the constants $k, \hat{k}$ in Eq. (29). There are three possible solutions:

1. $p$ linear near $-p_{0}$

2. $p$ linear near 0

3. $p$ linear near $p_{0}$

and $\phi<\phi_{\mathrm{c}}$ for each of these solutions. Solution 1 is shown in Fig. 1 in the right top for a $\mathrm{BaTiO}_{3}$ film for the same parameters as that used in the computational results of the full model shown on the left. We see that the agreement is good. We discard solution 2 as being unstable.

Let us now apply a field by applying the potentials $\phi_{0}=0, \phi_{L}=-V$ to the electrodes. For definiteness, assume that $V>0$, so that the electric field $E=V / L>0$. If $V$ is small, we look for a solution close to solution 1 above that only use in regime I. To do so, let $p^{-}, p^{+}$(Fig. 15) be close to $p_{0}$ and solve

$$
W\left(p^{+}\right)-W\left(p^{-}\right)=\rho_{0} V, \quad p^{+}-p^{-}=\rho_{0} L .
$$

We can now identify the constants $k, \hat{k}$ in Eqs. (28) and (29) by setting $p(0)=p^{-}$and $p(L)=p^{+}$. We can have a similar solution close to $p_{0}$. Returning to the solution near $-p_{0}$, we see that, as $V$ increases, $p^{-}, p^{+}$increase, until at some point, one or both are above the spinodal $-p_{s}$ and the solution becomes unstable. At this point, the solution near $p_{0}$ is the only stable solution. It is clear, therefore, that, in the limit of

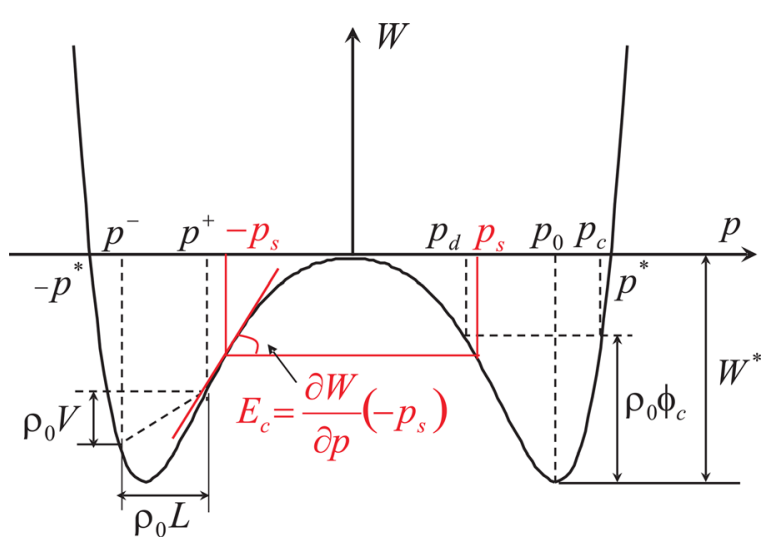

FIG. 15. The Devonshire-Landau energy and switching plot. 
very thin films, the switching field corresponds to the spinodal and is equal to the classical value $E_{c}$. As the thickness increases, the potential for switching increases, but at a rate slower than the thickness, so that the switching field decreases. In addition, in the limit of very thin films, $p^{-}, p^{+}$ simultaneously cross the spinodal $-p_{s}$ from $-p_{0}$ or cross the spinodal $p_{s}$ from $p_{0}$, resulting in a symmetric and classic switching field. This agrees with our computational results.

Intermediate films. Let $p_{c}-p_{d}<\rho_{0} L<2 p_{c}$. The analysis in this case is similar to that of Morozovska et al. ${ }^{10}$ Under zero applied field, as $L$ increases, solutions 1 and 3 above involve higher and higher potentials and eventually reach regime II. There are three possible solutions:

1. $p$ is linear, constant, linear near $-p_{0}$ as the potential $\phi$ increases to $\phi_{\mathrm{c}}$, remains constant, and then decreases to 0

$$
p(x)= \begin{cases}\rho_{0} x-p_{c} & 0 \leq x<\left(p_{c}-p_{0}\right) / \rho_{0}, \\ -p_{0} & \left(p_{c}-p_{0}\right) / \rho_{0} \leq x \leq L-\left(p_{0}-p_{d}\right) / \rho_{0}, \\ \rho_{0}(x-L)-p_{d} & L-\left(p_{0}-p_{d}\right) / \rho_{0}<x \leq L .\end{cases}
$$

\section{2. $p$ linear near 0}

3. $p$ is linear, constant, linear near $p_{0}$ as the potential $\phi$ increases to $\phi_{\mathrm{c}}$, remains constant, and then decreases to 0

$$
p(x)= \begin{cases}\rho_{0} x+p_{d} & 0 \leq x<\left(p_{0}-p_{d}\right) / \rho_{0}, \\ p_{0} & \left(p_{0}-p_{d}\right) / \rho_{0} \leq x \leq L-\left(p_{c}-p_{0}\right) / \rho_{0}, \\ \rho_{0}(x-L)+p_{c} & L-\left(p_{c}-p_{0}\right) / \rho_{0}<x \leq L .\end{cases}
$$

Solution 1 is shown in Fig. 1 in the right middle for a $\mathrm{BaTiO}_{3}$ film for the same parameters as that used in the computational results of the full model shown on the left. We see that the agreement is good. We discard solution 2 as being unstable.

Let us now apply a positive field by applying the potentials $\phi_{0}=0, \phi_{\mathrm{L}}=-V$ with $V>0$ to the electrodes. The situation is very similar to that discussed above for thin films if $\rho_{0} L<p_{c}$. If, however, $\rho_{0} L \geq p_{c}$, we see that solution 1 ceases to exist as $V$ increases beyond $W^{*} / \rho_{0}-\left|\phi_{c}\right|$. Thus, independent of thickness, the switching potential is equal to
$W^{*} / \rho_{0}-\left|\phi_{c}\right|$ as long as $\rho_{0} L \geq p_{c}$. This also means that the switching field decreases with film thickness. Recall the computational results (blue stars) we obtained from the full model in Fig. 7; we find a very good agreement. When the film thickness is beyond $p_{c} / \rho_{0}$, the switching potential under positive field (blue stars) is almost constant at $W^{*} / \rho_{0}-\left|\phi_{c}\right|$.

Further, as we point out before, the switching field/potential under negative field (red circle) is higher than the one under positive field. This asymmetry can be partially explained by looking at the right well in Fig. 15. We apply a negative field by applying the potentials $\phi_{0}=0, \phi_{L}=V$ with $V>0$ to the electrodes. As we increase $V$ slowly from 0 to $\phi_{c}$, the polarization at $x=0$ is fixed at $p_{d}$, while the polarization at $x=L$ slowly decreases from $p_{c}$ toward $p_{0}$. When $V$ is increased further, electrons start to flow into the film from the electrode at $x=L$, forming a negatively charged layer nearby, This, however, cannot be accounted by the simplified relation in Eq. (27); for more info, please refer to Xiao. ${ }^{29}$ and the polarization across the whole film starts to decrease until, at some point, the solution near the right well becomes unstable and the whole film suddenly switches to the left well. For the doping level we choose in computing Fig. $7, \phi_{c}=1.5 \mathrm{~V}$ is already slightly higher than $W^{*} / \rho_{0}-\left|\phi_{c}\right|=1.42 \mathrm{~V}$. Therefore, the switching potential under negative field is higher than the one under positive field. In addition, we notice that, similar to the positive case, the switching potential here is almost constant when the film thickness is beyond some value. For the negative field case here, this value is $\left(p_{c}-p_{d}\right) / \rho_{0}$, which is consistent with our analysis above.

Thick films. Let $2 p_{c}<\rho_{0} L$. As $L$ increases ever further with zero applied field, solutions 1 and 3 above remain the same, but the potential in solution 2 also now reaches $\phi_{c}$, so that one has now got to add regime II to this solution as well. So, the solutions are:

1. $p$ is linear, constant, linear near $-p_{0}$ as the potential $\phi$ increases to $\phi_{\mathrm{c}}$, remains constant, and then decreases to 0 , as in Eq. (36)

2. $p$ is linear, constant at $-p_{0}$, linear near 0 , constant at $p_{0}$ and linear as the potential $\phi$ increases to $\phi_{\mathrm{c}}$, remains constant, decreases and increases to $\phi_{c}$, remains constant, and then decreases to 0

$$
p(x)= \begin{cases}\rho_{0} x-p_{c} & 0 \leq x<\left(p_{c}-p_{0}\right) / \rho_{0} \\ -p_{0} & \left(p_{c}-p_{0}\right) / \rho_{0} \leq x \leq x^{*} \\ \rho\left(x-x^{*}\right)-p_{0} & x^{*}<x<x^{*}+2 p_{0} / \rho_{0} \\ p_{0} & x^{*}+2 p_{0} / \rho_{0} \leq x \leq L-\left(p_{c}-p_{0}\right) / \rho_{0} \\ \rho_{0}(x-L)+p_{c} & L-\left(p_{c}-p_{0}\right) / \rho_{0}<x \leq L\end{cases}
$$

where $x^{*}$ satisfies $\left(p_{c}-p_{0}\right) / \rho_{0}<x^{*}<L-\left(p_{c}+p_{0}\right) / \rho_{0}$. This is really a one parameter family of solutions, since $x^{*}$ can be chosen from a range of values.

3. $p$ is linear, constant, linear near $p_{0}$ as the potential $\phi$ increases to $\phi_{\mathrm{c}}$, remains constant, and then decreases to 0 , as in Eq. (37).
Solution 1 is shown in Fig. 1 in the right bottom for a $\mathrm{BaTiO}_{3}$ film for the same parameters as that used in the computational results of the full model shown on the left. We see that the agreement is good, except the potential is cut-off in the middle. We discard solution 2 as being unstable. 
As to the switching behavior for thick films, the situation is very similar to intermediate films with $L \geq p_{c} / \rho_{0}$ under positive field and $L \geq\left(p_{c}-p_{d}\right) / \rho_{0}$ under negative field. Both switching potentials are kept constant, thus the switching fields continue to decrease with thickness. This is again consistent with our computational results.

\section{B. High doping}

We assume that $\rho_{0}\left|\phi_{c}\right|>W^{*}$ in this section. Let $\pm p_{c}$ $\left(p_{c}>p_{0}\right)$ be the points, as shown in Fig. 14(b), which satisfy

$$
W\left( \pm p_{c}\right)=W\left(p_{0}\right)+\rho_{0}\left|\phi_{c}\right| .
$$

We can have three types of films, depending on thickness. For the material parameters and doping level we consider in Fig. 3, films with $L<67.8 \mathrm{~nm}$ belong to the thin film regime, films with $L>101 \mathrm{~nm}$ belong to the thick film regime, and the intermediate film regime includes films with thickness in between.

Thin films. Let $\rho_{0} L \leq 2 p_{0}$. We begin with the case of zero applied field. It is possible now to find solutions which only use regime I. We use the boundary conditions to find the constants $k, \hat{k}$ in Eq. (29). There are three possible solutions:

1. $p$ linear near $-p_{0}$

2. $p$ linear near 0

3. $p$ linear near $p_{0}$

and $\phi<\phi_{\mathrm{c}}$ for each of these solutions. There are no other solutions, as the potential can never get high enough to $\phi_{c}$. Solution 1 is shown in Fig. 3 in the right top for a $\mathrm{BaTiO}_{3}$ film for the same parameters as that used in the computational results of the full model shown on the left. We see that the agreement is good. We discard solution 2 as being unstable. Two thicknesses are considered in Fig. 3 for the thin film regime, one with $L=10 \mathrm{~nm}$ and one with $L=60 \mathrm{~nm}$. The profile of the 10 -nm case is very similar to the one with the same thickness, but lower doping considered earlier in Fig. 1. The 60-nm case, however, has a slightly different profile, reflecting the fact that the polarization is ranging from negative to positive domain. The situation with applied field, including the switching field, is similar to the lower doping cases.

Intermediate films. Let $2 p_{0}<\rho_{0} L \leq 2 p_{c}$. In the case of zero applied field, as $L$ increases beyond $2 p_{0} / \rho_{0}$, the solutions near $\pm p_{0}$ are lost, as the potential is unable to climb back to zero. Therefore, we are left with a unique solution:

1. $p$ linear near 0

This solution is shown in the right column in Fig. 3 for a $\mathrm{BaTiO}_{3}$ film with $L=100 \mathrm{~nm}$ for the same parameters as that used in the computational results of the full model shown on the left. The agreement between the full and the approximate models is again very good.

Let us now apply a negative field by applying the potentials $\phi_{0}=0, \phi_{L}=V$ with $V>0$ to the electrodes. We continue to have a unique solution for a moderate $V$ and start to have two solutions when $V$ increases further. When the original solution becomes unstable, the polarization at $x=L$ jumps from positive value to negative value, while the polarization at $x=0$ hardly changes. If we apply a positive field by applying the potentials $\phi_{0}=0, \phi_{L}=-V$ with $V>0$ to the electrodes, we continue to have a unique solution with increasing $V$. When $V$ increases further, passing a point $(1.3 \mathrm{~V})$ when the approximation in Eq. (27) is no longer valid, ${ }^{29}$ huge positive charges start to accumulate near the electrode at $x=L$, pushing the polarization nearby to a superficially high level, while the polarization at $x=0$ hardly changes.

Thick films. Let $2 p_{c} \leq \rho_{0} L$. In the case of zero applied field, as $L$ increases further, the potential increases to $\phi_{c}$, so that one has to use regime II. The solution now is

1. $p$ linear, constant at $-p_{0}$, linear around 0 , constant at $p_{0}$, and linear as the potential increases from zero to $\phi_{c}$, remains constant, decreases and increases back to $\phi_{c}$, remains constant, and decreases back to zero

$$
p(x)= \begin{cases}\rho_{0} x-p_{c} & 0 \leq x<\left(p_{c}-p_{0}\right) / \rho_{0} \\ -p_{0} & \left(p_{c}-p_{0}\right) / \rho_{0} \leq x \leq x^{*} \\ \rho_{0}\left(x-x^{*}\right)-p_{0} & x^{*}<x<x^{*}+2 p_{0} / \rho_{0} \\ p_{0} & x^{*}+2 p_{0} / \rho_{0} \leq x \leq L-\left(p_{c}-p_{0}\right) / \rho_{0} \\ \rho_{0}(x-L)+p_{c} & L-\left(p_{c}-p_{0}\right) / \rho_{c}<x \leq L\end{cases}
$$

where $x^{*}$ satisfies $\left(p_{c}-p_{0}\right) / \rho_{0}<x^{*}<L-\left(p_{c}+p_{0}\right) / \rho_{0}$. We now have a solution with "tail-to-tail" domains. Further, notice that this is really a one parameter family of solutions, since $x^{*}$ can be chosen from a range of values. This solution is shown in Fig. 3 in the right bottom for a $\mathrm{BaTiO}_{3}$ film for the same parameters as that used in the computational results of the full model shown on the left. We see that the agreement is good.

Suppose we apply a positive field with $\phi_{0}=0, \phi_{\mathrm{L}}=-V$ for some $V>0$. Let $p_{V}>0$ be the solution to $W( \pm$ $\left.p_{V}\right)=\rho_{0} V+W\left(p_{c}\right)$. For $V$ small enough so that $p_{V}+p_{c}<\rho_{0} L$, we have a one-parameter family of solutions, 


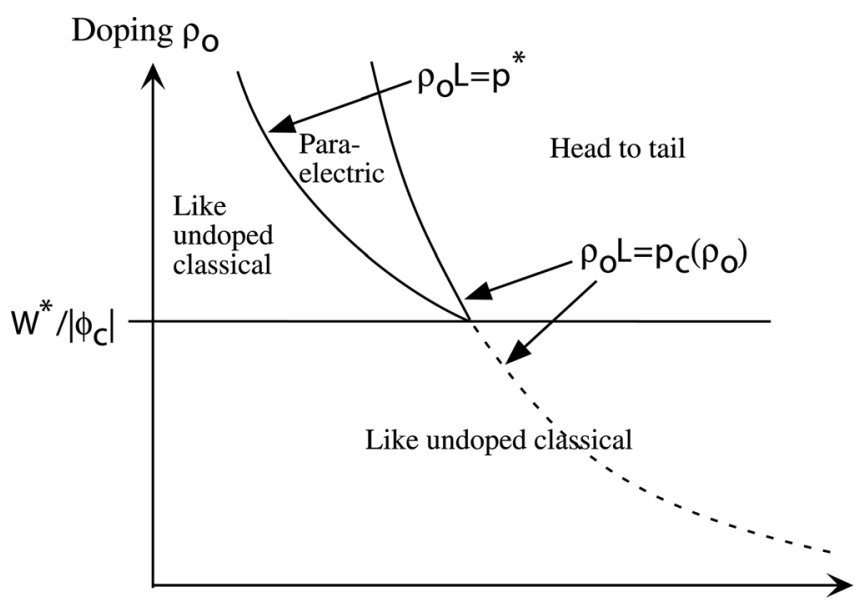

Film Thickness L

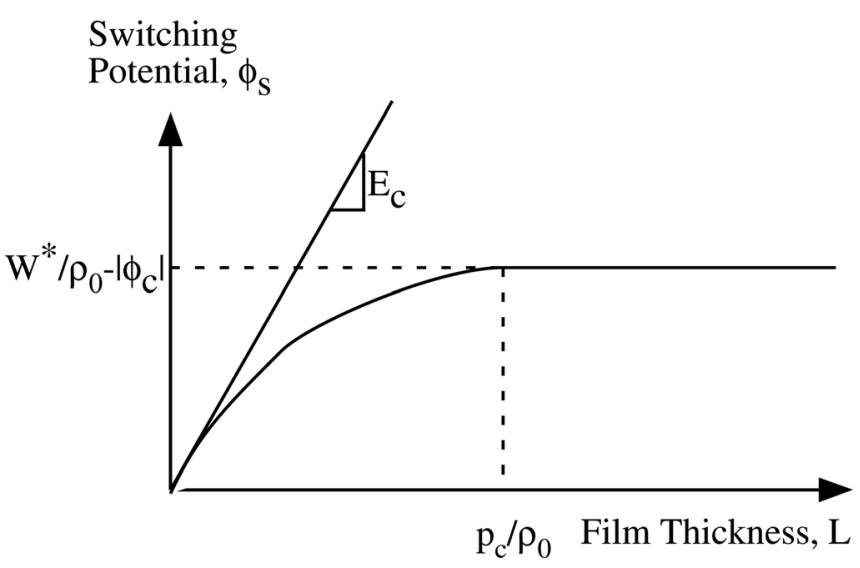

FIG. 17. The potential required for switching a film with low doping.

FIG. 16. Behavior of films of different thickness and doping level.

$$
p(x)= \begin{cases}\rho_{0} x-p_{c} & 0 \leq x<\left(p_{c}-p_{0}\right) / \rho_{0} \\ -p_{0} & \left(p_{c}-p_{0}\right) / \rho_{0} \leq x \leq x^{*} \\ \rho_{0}\left(x-x^{*}\right)-p_{0} & x^{*}<x<x^{*}+2 p_{0} / \rho_{0} \\ p_{0} & x^{*}+2 p_{0} / \rho_{0} \leq x \leq L-\left(p_{V}-p_{0}\right) / \rho_{0} \\ \rho_{0}(x-L)+p_{V} & L-\left(p_{V}-p_{0}\right) / \rho_{0}<x \leq L\end{cases}
$$

It is easy to verify that the energy associated with this solution is independent of $x^{*}$. This implies that, as long as $V$ is small enough so that $p_{V}+p_{c}<\rho_{0} L$, the film feels no energetic driving force to switch. In effect, the high doping level and ample length create enough charges to "screen" the effect of the field. Notice that, for any $V$, the part of the film in regime II has length $L-\left(p_{V}+p_{c}\right) / \rho_{0}$. Therefore, as $V$ increases to reach the point that $p_{V}+p_{c}=\rho_{0} L$, the part of the film in regime II shrinks to zero and we have a unique solution. However, for the case we consider in Fig. 10 and also in Fig. 13, before $V$ even reaches that point, a very thin layer with large positive charges appears near the electrode at $x=L$ (see the insets in Fig. 13 for point $\mathrm{F}$ ) for the reason that we discussed in the intermediate case. This thin layer at $x=L$ pushes the polarization nearby to a superficially high level and keeps the polarization at $x=0$ almost unchanged.

If we now apply a negative field with $\phi_{0}=0, \phi_{L}=V$ for some $V>0$. And $p_{V}>0$ is the solution to $W\left( \pm p_{V}\right)=W\left(p_{c}\right)-\rho_{0} V$. Assume $V$ is small enough so that $p_{V}>p_{0}$, we still have a one-parameter family of solutions the same as Eq. (41). As $V$ increases, the polarization at $x=L$ decreases toward $p_{0}$, while the left part near $x=0$ is kept unchanged. When $V$ further increases and passes $\phi_{c}$ (point B in Fig. 10), the solution becomes unstable and the polarization at $x=L$ suddenly jumps from positive to negative value, while the polarization at $x=0$ hardly changes. Therefore, the hysteresis loop formed by the effective polarization at $x=L$ and the electric field resides entirely in the negative domain, exactly as shown in Fig. 10.

\section{SUMMARY AND CONCLUSIONS}

We have presented a theory for ferroelectrics, which are also wide bandgap semiconductors. The theory does not make any a priori assumption about either the space charge distribution or the polarization profile. We have studied a metal-ferroelectric-metal capacitor configuration, where the ferroelectric is n-type doped in some detail. Our main result is summarized in Fig. 16. We can identify a critical doping level, which depends on the energy barrier in the Landau energy and the build-in potential $\phi_{c}$, which is decided by the electronic structures of both the film and the electrodes. When the doping level is below this critical value, the behavior of the films is almost classical. We see a depleted region, which extends through the film when the film thickness is very small, but is confined to two boundary layers near the electrodes for large film thickness. When the doping level is higher than the critical value, the behavior is classical for only very thin films. Thicker films at this doping level are forced into a tail-to-tail configuration with three depletion layers, lose their ferroelectricity, and may thus be described as nonlinear dielectric or paraelectric.

For films which are doped below the critical level, we show that the field required for switching starts out at the classical coercive field for very thin films, but gradually decreases as indicated in Fig. 17. In fact, the potential required for switching is constant beyond a certain thickness, as shown in Fig. 7(b). The depletion boundary layers provide a natural mechanism for the nucleation of the oppositely 
polarized domain. However, we also notice the limitation of our 1D model, since domain patterns and switchings are absent here. Without domain patterns, the switching process is not realistic and complete. However, the switching behavior we obtain here nevertheless provides us very fresh and surprisingly useful insights in understanding the switching behavior of defect films and motivates us for future work on more complex models.

Finally, the importance in the choice of the electrode is highlighted by the fact that the width of depletion layers depends on $\phi_{c}$, which, in turn, depends on the work function of the electrode. The higher the work function, the higher the build-in potential, and the wider the depletion layers, the harder the film subject to failure, ${ }^{15}$ and thus the better the behavior of the films.

\section{ACKNOWLEDGMENTS}

It is our pleasure to acknowledge Jim Scott (Cambridge) for bringing this problem to our attention and many delightful discussions. This work started when V.B.S. and K.B. were visiting the Isaac Newton Institute, Cambridge and we are grateful for their hospitality. We gratefully acknowledge the support of the National Science Foundation (K.B. and Y.X.: MRSEC.FNP-1.2-NSF.CSEM) and the Army Research Office (K.B. and Y.X.: DAAD19-01-1-0517 and W911NF0810269; V.B.S.: W911NF-11-1-0171).

${ }^{1}$ J. F. Scott, Ferroelectric Memories (Springer, 2000).

${ }^{2}$ V. Fridkin, Ferroelectric Semiconductors (Consultants Bureau, 1980).

${ }^{3}$ A. Bratkovsky and A. Levanyuk, Phys. Rev. B 61, 15042 (2000).
${ }^{4}$ A. Tagantsev, C. Pawlaczyk, K. Brooks, M. Landivar, E. Colla, and N. Setter, Integr. Ferroelectr. 6, 309 (1995).

${ }^{5}$ L. Pintilie and M. Alexe, J. Appl. Phys. 98, 124103 (2005).

${ }^{6}$ Y. Watanabe, Appl. Surf. Sci. 132, 610 (1998).

${ }^{7}$ Y. Watanabe, Phys. Rev. B 57, 789 (1998).

${ }^{8}$ P. Zubko, D. J. Jung, and J. F. Scott, J. Appl. Phys. 100, 114112 (2006).

${ }^{9}$ L. Baudry, J. Appl. Phys. 86, 1096 (1999).

${ }^{10}$ A. N. Morozovska, E. A. Eliseev, S. V. Svechnikov, A. D. Krutov, V. Y. Shur, A. Y. Borisevich, P. Maksymovych, and S. V. Kalinin, Phys. Rev. B 81, 205308 (2010).

${ }^{11}$ M. Y. Gureev, A. K. Tagantsev, and N. Setter, Phys. Rev. B 83, 184104 (2011).

${ }^{12}$ E. A. Eliseev, A. N. Morozovska, G. S. Svechnikov, V. Gopalan, and V. Y. Shur, Phys. Rev. B 83, 235313 (2011).

${ }^{13}$ P. Maksymovych, J. Seidel, Y. H. Chu, P. Wu, A. P. Baddorf, L.-Q. Chen, S. V. Kalinin, and R. Ramesh, Nano Lett. 11, 1906 (2011).

${ }^{14}$ P. Suryanarayana and K. Bhattacharya, J. Appl. Phys. 111, 034109 (2012).

${ }^{15}$ Y. Xiao, V. B. Shenoy, and K. Bhattacharya, Phys. Rev. Lett. 95, 247603 (2005).

${ }^{16}$ Y. Xiao and K. Bhattacharya, Arch. Rational Mech. Anal. 189, 59 (2008).

${ }^{17}$ S. M. Sze, Physics of Semiconductor Devices, 2nd ed. (Wiley, 1981).

${ }^{18}$ F. Jona and G. Shirane, Ferroelectric Crystals (Dover, New York, 1993).

${ }^{19}$ M. Drougard and E. Huibregtse, IBM J. Res. Dev. 1, 318 (1957).

${ }^{20}$ A. Moulson and J. Herbert, Electroceramics: Materials, Properties, Applications, 2nd ed. (Wiley, 2003).

${ }^{21}$ M. Renardy and R. Rogers, An Introduction to Partial Differential Equations, 2nd ed. (Springer, 2004).

${ }^{22}$ L. Baudry and J. Tournier, J. Appl. Phys. 97, 024104 (2005).

${ }^{23}$ G. Arlt and H. Neumann, Ferroelectrics 87, 109 (1988).

${ }^{24}$ D. Hall and M. Ben-Omran, J. Phys.: Condens. Matter 10, 9129 (1998).

${ }^{25}$ W. Warren, D. Dimos, G. Pike, B. Tuttle, M. Raymond, R. Ramesh, and J. Evans, Appl. Phys. Lett. 67, 866 (1995).

${ }^{26}$ S. Okamura, S. Miyata, Y. Mizutani, T. Nishida, and T. Shiosaki, Jpn. J. Appl. Phys. 38, 5364 (1999).

${ }^{27}$ L. Zheng, C. Lin, W. Xu, and M. Okuyama, J. Appl. Phys. 79, 8634 (1996).

${ }^{28}$ M. Dawber, K. Rabe, and J. Scott, Rev. Mod. Phys. 77, 1083 (2005).

${ }^{29}$ Y. Xiao, Ph.D. dissertation, California Institute of Technology, 2004. 